\title{
Interoperability of Contact and Contactless Fingerprints Across Multiple Fingerprint Sensors
}

\author{
Brady M. Williams \\ West Virginia University, bwwilliams@mix.wvu.edu
}

Follow this and additional works at: https://researchrepository.wvu.edu/etd

Part of the Other Electrical and Computer Engineering Commons

\section{Recommended Citation}

Williams, Brady M., "Interoperability of Contact and Contactless Fingerprints Across Multiple Fingerprint Sensors" (2021). Graduate Theses, Dissertations, and Problem Reports. 10159.

https://researchrepository.wvu.edu/etd/10159

This Thesis is protected by copyright and/or related rights. It has been brought to you by the The Research Repository @ WVU with permission from the rights-holder(s). You are free to use this Thesis in any way that is permitted by the copyright and related rights legislation that applies to your use. For other uses you must obtain permission from the rights-holder(s) directly, unless additional rights are indicated by a Creative Commons license in the record and/ or on the work itself. This Thesis has been accepted for inclusion in WVU Graduate Theses, Dissertations, and Problem Reports collection by an authorized administrator of The Research Repository @ WVU. For more information, please contact researchrepository@mail.wvu.edu. 


\title{
Interoperability of Contact and Contactless Fingerprints Across Multiple Fingerprint Sensors
}

\author{
Brady Williams \\ Thesis submitted to the \\ Benjamin M. Statler College of Engineering and Mineral Resources \\ at West Virginia University \\ in partial fulfillment of the requirements \\ for the degree of
}

Master of Science

in

Computer Science

Jeremy Dawson, Ph.D., Chair

Nasser Nasrabadi, Ph.D.

Matthew Valenti, Ph.D.

Lane Department of Computer Science and Electrical Engineering

Morgantown, West Virginia

2021

Keywords: Fingerprints, Fingerprint Matching, Contactless Fingerprint, Interoperability

Copyright $@ 02021$ Brady Williams 


\begin{abstract}
Interoperability of Contact and Contactless Fingerprints Across Multiple Fingerprint Sensors
\end{abstract}

Brady Williams

Contactless fingerprinting devices have grown in popularity in recent years due to speed and convenience of capture. Also, due to the global COID-19 pandemic, the need for safe and hygienic options for fingerprint capture are more pressing than ever. However, contactless systems face challenges in the areas of interoperability and matching performance as shown in other works. In this paper, we present a contactless vs. contact interoperability assessment of several contactless devices, including cellphone fingerphoto capture. During the interoperability assessment, the quality of the fingerprints was considered using the NBIS NFIQ software with the contact-based fingerprint performing the best overall as expected. In addition to evaluating the match performance of each contactless sensor, this paper presents an analysis of the impact of finger size and skin melanin content on contactless match performance. AUC results indicate that contactless match performance of the newest contactless devices is reaching that of contact fingerprints. In addition, match scores indicate that, while not as sensitive to melanin content, contactless fingerprint matching may be impacted by finger size. 


\section{Contents}

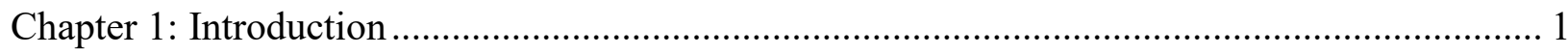

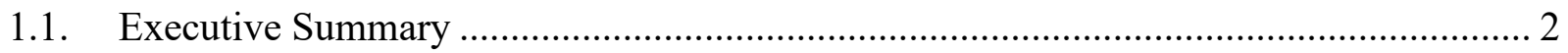

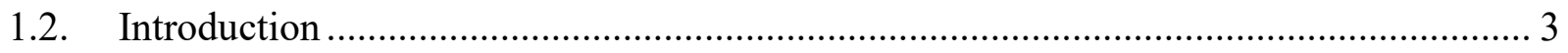

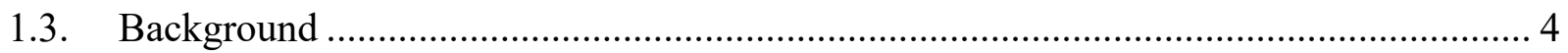

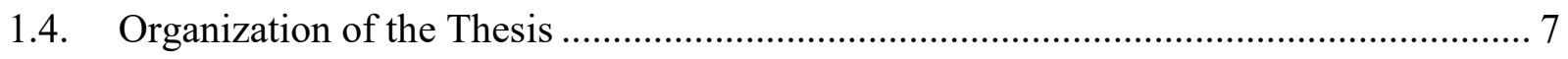

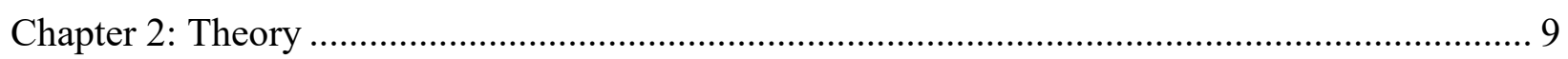

2.1. Contact and Contactless Sensor Hardware ................................................................... 10

2.1.1. Frustrated Total Internal Reflection (FTIR) ...................................................... 10

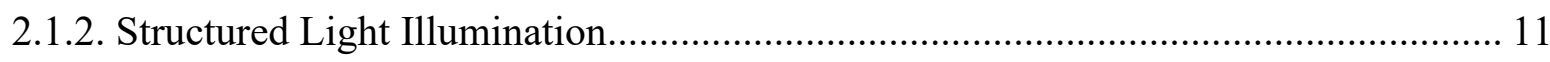

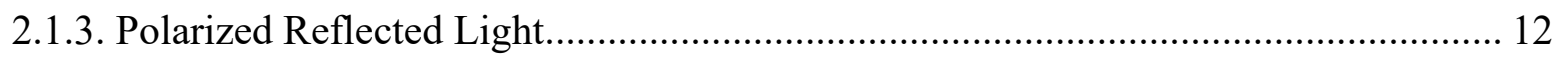

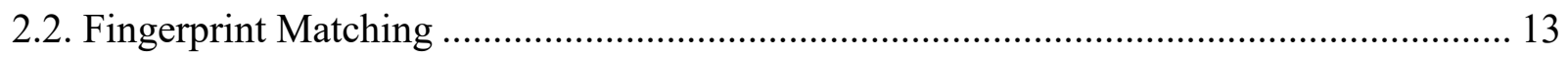

2.2.1. Fingerprint Matching and Recognition ...................................................................... 13

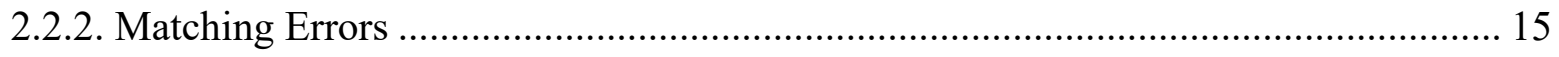

2.2.3. Receiver Operating Characteristic Curves ............................................................. 15

2.2.4. NIST Fingerprint Image Quality (NFIQ) ……………............................................... 16

Chapter 3: Dataset Details and Matching Experiments ................................................................ 17

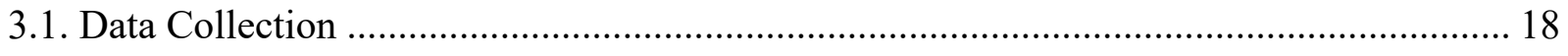

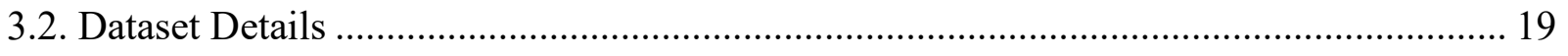

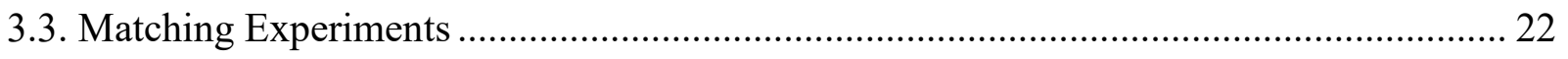

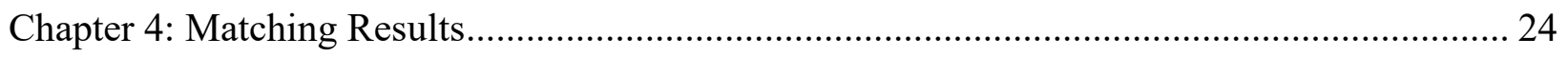

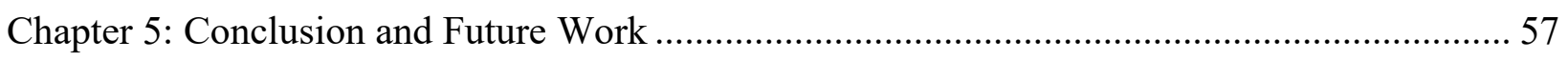

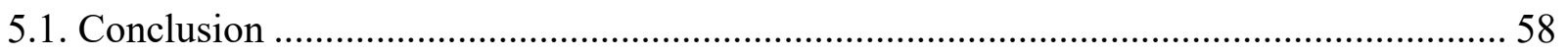

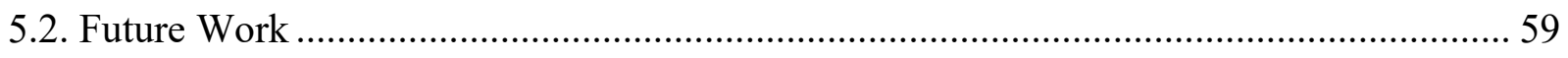

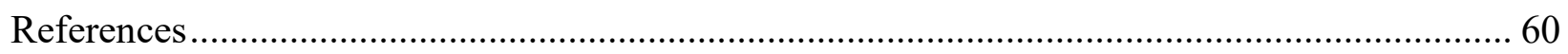




\section{List of Tables}

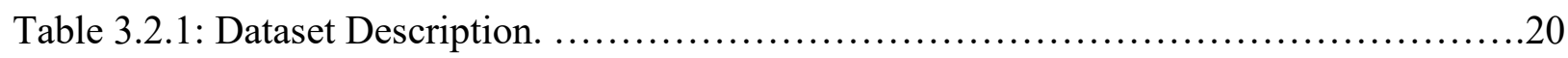

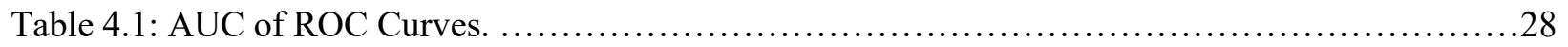




\section{List of Figures}

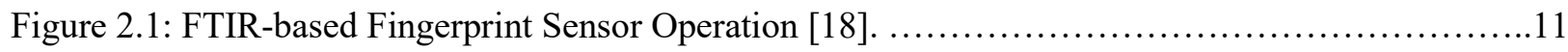

Figure 2.2: Example of Structured Light Illumination Applied to a Finger [20]. ....................12

Figure 2.3: General Block Diagram for a Fingerprint Identification System [28] .....................13

Figure 2.4: Typical Minutiae Matching Algorithm [25] ...................................... 15

Figure 2.5: Example ROC Curve. ...................................................... 16

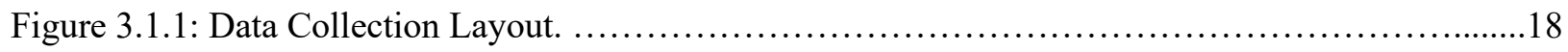

Figure 3.1.2: Data Collection in Progress Photo. ........................................... 19

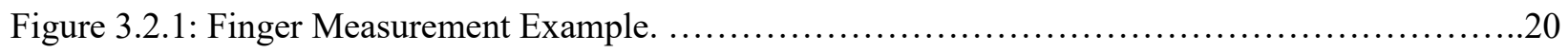

Figure 3.2.2: Participant Demographics by Gender and Ethnicity. ..............................2

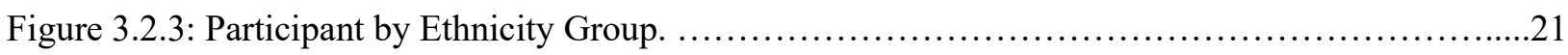

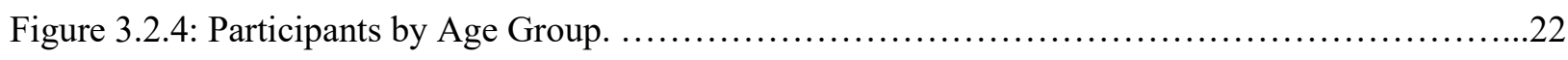

Figure 3.3.1: Step-by-Step Preprocessing for Cellphone-Based Fingerphotos Showing (a) Original (b)

Grayscale (c) Adaptive Histogram Equalization (d) Inverted. ..................................... 23

Figure 4.1: Receiver Operating Characteristic for contact and contactless fingerprint devices against

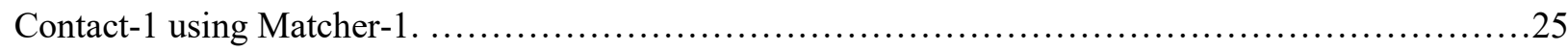

Figure 4.2: Receiver Operating Characteristic for contact and contactless fingerprint devices against

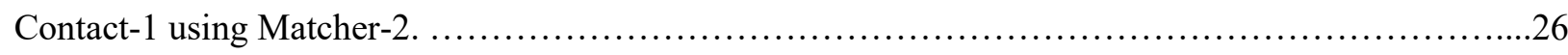

Figure 4.3: Receiver Operating Characteristic for contact and contactless fingerprint devices against

Contact-1 using Matcher-3. .......................................................... 27

Figure 4.4: NFIQ Score Distribution for All Devices. ......................................29

Figure 4.5: Comparison of the distribution of mated match scores for each device using Matcher-1. .......30

Figure 4.6: Comparison of the distribution of mated match scores for each device using Matcher-2. ......31

Figure 4.7: Comparison of the distribution of mated match scores for each device using Matcher-3. ......32

Figure 4.8: Comparison of the distribution of mated match scores based on melanin amount using probes

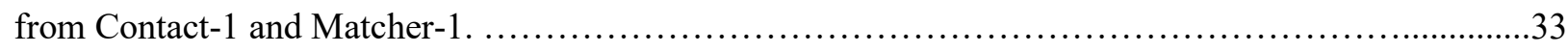


Figure 4.9: Comparison of the distribution of mated match scores based on melanin amount using probes

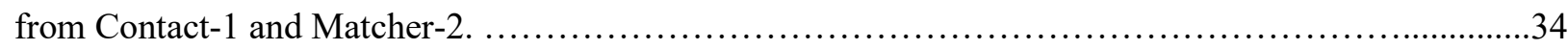

Figure 4.10: Comparison of the distribution of mated match scores based on melanin amount using probes

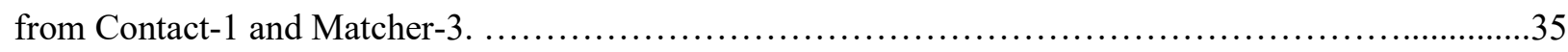

Figure 4.11: Comparison of the distribution of mated match scores based on melanin amount using probes

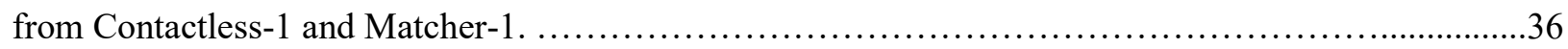

Figure 4.12: Comparison of the distribution of mated match scores based on melanin amount using probes

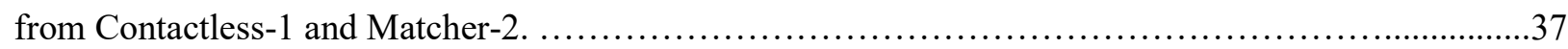

Figure 4.13: Comparison of the distribution of mated match scores based on melanin amount using probes

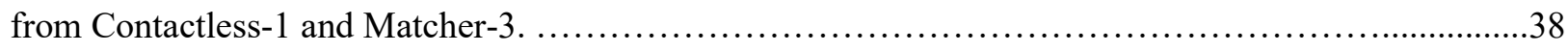

Figure 4.14: Comparison of the distribution of mated match scores based on melanin amount using probes from Contactless-2 and Matcher-1. .........................................................

Figure 4.15: Comparison of the distribution of mated match scores based on melanin amount using probes

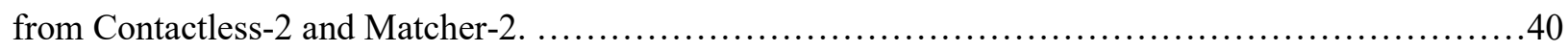

Figure 4.16: Comparison of the distribution of mated match scores based on melanin amount using probes from Contactless-2 and Matcher-3. ................................................... 41

Figure 4.17: Comparison of the distribution of mated match scores based on melanin amount using probes

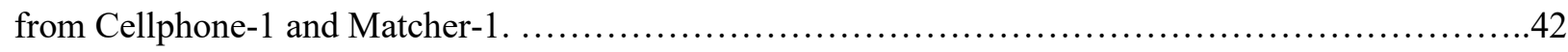

Figure 4.18: Comparison of the distribution of mated match scores based on melanin amount using probes

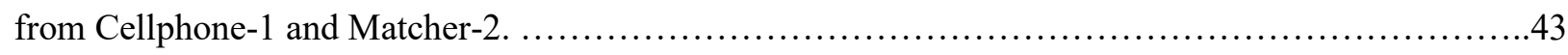

Figure 4.19: Comparison of the distribution of mated match scores based on melanin amount using probes from Cellphone-1 and Matcher-3. .................................................... 44

Figure 4.20: Comparison of the distribution of mated match scores based on middle finger width using probes from Contact-1 and Matcher-1...................................................45

Figure 4.21: Comparison of the distribution of mated match scores based on middle finger width using

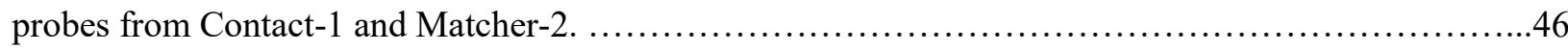

Figure 4.22: Comparison of the distribution of mated match scores based on middle finger width using probes from Contact-1 and Matcher-3. 
Figure 4.23: Comparison of the distribution of mated match scores based on middle finger width using probes from Contactless-1 and Matcher-1.

Figure 4.24: Comparison of the distribution of mated match scores based on middle finger width using probes from Contactless-1 and Matcher-2.

Figure 4.25: Comparison of the distribution of mated match scores based on middle finger width using probes from Contactless-1 and Matcher-3.

Figure 4.26: Comparison of the distribution of mated match scores based on middle finger width using probes from Contactless-2 and Matcher-1.

Figure 4.27: Comparison of the distribution of mated match scores based on middle finger width using probes from Contactless-2 and Matcher-2.

Figure 4.28: Comparison of the distribution of mated match scores based on middle finger width using probes from Contactless-2 and Matcher-3.

Figure 4.29: Comparison of the distribution of mated match scores based on middle finger width using probes from Cellphone-1 and Matcher-1.

Figure 4.30: Comparison of the distribution of mated match scores based on middle finger width using probes from Cellphone-1 and Matcher-2.

Figure 4.31: Comparison of the distribution of mated match scores based on middle finger width using probes from Cellphone-1 and Matcher-3. .56 


\section{Chapter 1: \\ Introduction}




\subsection{Executive Summary}

The use of fingerprints for biometric identification is a well-studied and researched area of biometrics. The uniqueness of fingerprints, even among those closely related and in large populations, along with their general acceptance and relative ease of collection has led to widespread usage in many scenarios. Over time, the process for fingerprint collection has evolved beyond the early inked fingerprinting methods. These changes in methods include digitizing inked fingerprints, contact-based livescan fingerprints, and contactless-based fingerprints. With the advent of contactless fingerprinting techniques, new challenges in interoperability became apparent with differences in image quality and detail, as well as differences in the inherent geometry of the captures themselves.

Increased interest in contactless fingerprinting has grown due to the convenient nature and speed of the capture as well as the health and safety benefits that come with not having to physically interact with the capture device. The convenience of this capture method comes with a new set of drawbacks and issues, as described in other published works that will be explored further in the first chapter. This thesis will present an analysis of the interoperability of contactless fingerprints with traditional contact-based fingerprints. This study will explore common contactless-based solutions and a cellphone app designed to capture images using the built-in camera in the cellphone it is installed on. The interoperability performance of the contactless methods will be evaluated by comparing the match scores of the contactless-based fingerprints to their contact-based counterparts. Along with the matching performance, a brief study into the quality of the output fingerprint images, performed using the NFIQ software available in the NBIS software package from the National Institute of Standards and Technology (NIST), is presented. Beyond the general interoperability study, this work also explored the effects of skin reflectance value or the finger size on the matching interoperability of contactless fingerprints.

The results of these studies show that the contactless fingerprints used exhibit variable match performance, the device labeled Contactless-2 matching closely to the contact-to-contact baseline. The performance of all devices shows that the field of contactless fingerprints continues to improve but has not fully reached the standards of contact fingerprints yet. In terms of the skin reflectance study, the results show no noticeable effect on the matching results due to 
the skin reflectance when compared between all devices including, the contact baseline. For the exploration of finger size, there was a noticeable affect wherein Contactless-1 and Contactless- 2 tended to perform better with larger fingers when using Matcher-1. This effect was not present for Cellphone-1 and was not present on either of the other matchers.

\subsection{Introduction}

The use of fingerprints for identification and verification has been commonplace for many years in commercial, consumer, and government applications. Fingerprint matching is performed using the minutiae present in all fingerprints that are extracted from the ridge and valley features of the fingerprint [1].

As technology has advanced, so have the methods for fingerprint collection. From inked fingerprints on paper, to contact-based livescan fingerprinting, to contactless fingerprint imaging, while the image capture process may be different, the resulting fingerprint must still be interoperable in matching against legacy contact galleries. Traditional contact-based digital fingerprints are recorded optically by physically placing the finger or hand on a device, typically against a glass platen, or electronically based on the conductivity of the skin. These contactbased methods impart some degree of elastic deformation on the finger, and consequently, to the ridges of the fingerprint. Contactless fingerprints pose an interoperability problem as they lack the elastic deformation caused by pressing the finger against the capture device [2]. In addition, because they are essentially created from fingerphotos, contactless fingerprints may contain high degrees of photometric distortion that, in addition to the lack of elastic deformation, may further reduce matching interoperability [2] [3] [4]. The quality of the fingerprints acquired from these devices can be measured using the NFIQ software which analyzes the fingerprints based on how well they can provide the minutiae used in traditional fingerprint matching [2].

The ubiquitous nature of smartphone cameras and their use in multibiometric capture, as well as the emergence of COVID-19 as a major health crisis, have driven the need for fast, hygienic capture of contactless fingerprints, making studies of contactless fingerprint imaging interoperability even more necessary. The overall goal of the work presented here is to evaluate the interoperability of multiple contactless fingerprints when matched against contact fingerprints collected from the same individuals. In addition to this baseline interoperability 
analysis, physiological factors such as skin color and finger size are evaluated to determine their impact on contactless fingerprint matching.

The contributions of this research effort are: 1) a quantification of the interoperability of contactless fingerprints from two contactless devices and one cellphone-based fingerprint collection method against a traditional contact-based digital fingerprinting device including an analysis of the quality of the fingerprints captured, 2) a measurement of the effect of hand size on the overall matching performance of fingerprints, and 3) an exploration of the effect of skin color measured by skin reflectance on the overall matching interoperability and matching performance of contactless-based fingerprints. The results presented here provide critical insight into the application of contactless fingerprinting systems in a variety of biometric scenarios.

\subsection{Background}

Two forms of contactless fingerprints were examined in this effort. The first form is contactless fingerprints captured from a standalone kiosk-type sensor that images the finger when in the field of view of the device (see, e.g., [2], [3], [5], [6], [7]). The second form is contactless fingerprints that are captured using a cellphone app that employs the built-in camera to capture fingerphotos [2]. The images from the cellphone then undergo processing to create a binarized or grayscale fingerprint image that are representative of the original fingerphoto captured from the cellphone camera. While these images look visually different than those from traditional fingerprinting methods, they still aim to provide the expected minutiae from the fingerprints that is critical for traditional matching techniques. However, the minutiae simply being present does not guarantee the quality of the minutiae or fingerprint image as a whole. In the vein of matching quality contactless fingerprints have performed worse than the traditional contact-based counterparts as shown in [2].

To evaluate the interoperability of these fingerprints, two commercial 'black-box' fingerprint matchers will be used, along with one open-source matcher. These three solutions rely on minutiae correspondence as the primary method for matching [1], [8]. This involves extracting the minutiae from each fingerprint as a set of features that are then compared to find the match score. The two commercial off the shelf (COTS) solutions provide everything necessary to perform the fingerprint matching by being able to convert the images if a file type change is necessary, extract the minutiae from the images that are passed for the gallery and probes and 
generate the templates used in the matching process, and finally by performing the matching itself based on these templates used. Both solutions use proprietary template formats that the end user does not have open access to, along with the fingerprint matching algorithms that are hidden from the end user as well. The open-source matcher works in the same way to perform the matching, but the template generation and matching are performed by different applications. The first generates minutiae files, in the same way the COTS matchers use templates, that provide the location and direction of the detected minutiae. The second program looks at the minutiae files and performs the matching based on these. At its core this solution does not provide image manipulation within these two programs, however, other software can be had from the same source to complete any necessary file conversions.

While the use and capture of contactless fingerprints are relatively new developments, there has been work done to evaluate and use this form of capture with contact-based fingerprint galleries. NIST has provided recommendations on evaluation of contactless fingerprint devices [2]. This study outlines the considerations necessary for proper capture of contactless fingerprints, and how these differ from traditional fingerprints. This study also considers the standards previously determined for contact-based fingerprint capture that are used in the certification of fingerprint capture and scanning devices [2], [9]. The study also focuses in on issues that are more specific to contactless capture such as blur in the images as a result of hand movement either necessary or not to the capture process. Studies such as the one described in [10] have been performed in a similar area to consider the use of the NIST NFIQ 2.0 method of fingerprint evaluation on contactless fingerprint to provide a quantitative measure of their usability. While, this study focuses on optimizing fingerprint post-processing for increased NFIQ score performance, it also provides insight into the reliability of the NFIQ scores for evaluating how well a fingerprint will perform in traditional matching scenarios.

In addition to best practices for contactless fingerprint applications, there have been other studies into the interoperability of contactless and contact-based fingerprints [3], [11], [12]. These studies have shown the challenges and variability issues that are common when collecting contactless fingerprints. Some of these challenges include differences in geometric distortion from the lack of pressure applied during capture, variability in the quality of the prints due to being converted from visual images, and differences in the clarity of ridges in the prints due to 
the same conversion process due to the changed perspective in recording the visual images that are the starting point.

Other studies have been performed to examine the use of deep learning for both matching and closing the interoperability gap between contact and contactless fingerprints. Convolutional neural networks $(\mathrm{CNN})$ that use preprocessed versions of both the contact and contactless prints to perform the matching were demonstrated in [13]. Here, the preprocessed fingerprints are passed into a Siamese CNN to provide 1:n match scores. An alternative CNN-based method presented in [14] uses a pair of CNNs to first find the amount of warp on the contactless fingerprint image, and then use that warp parameter to generate a new version of the contactless fingerprint that is representative of a contact-based fingerprint of the same finger. The study in [15] does not involve deep learning but is relevant as a source for quantifying the warp that contact-based fingerprints have due to the elastic deformation. This can then also be considered inversely as the warp the contactless prints do not have, but that needs to be accounted for when comparing the two types of fingerprint images.

Because of the nascent nature and methodology of contactless fingerprinting, physiological features that have little to no impact on contact fingerprint collection, such as finger size and skin color, may negatively impact contactless fingerprint interoperability. However, these features have received little evaluation in the literature in this context. Hand geometry features have been used in biometric verification applications. Hand geometry biometrics rely on the hand shape and various parameters of the hand's size as the features to be extracted and compared [16]. Relating to contactless fingerprints, the variation in finger sizes from person to person may have an impact on contactless matching performance when compared to a gallery of contact images. The finger size referenced can be measured from the fingers including their length between joints and width. The width in this case would be the width when in the same orientation as when contactless fingerprints would be captured.

Skin tone, also referred to as skin reflectance, is an important factor to consider in face detection and recognition [17]. Variations in skin reflectance, as well as differences in lighting, in facial imagery can have a major effect on the outcome of facial recognition and matching. This is typically not an issue when it comes to contact fingerprints because the method of acquisition is not photo-based. Contactless fingerprints, however, rely on fingerphotos to obtain 
the ridge and valley information of the fingerprint. As with facial images, variation in skin reflectance could have a significant effect on the matching accuracy of the fingerprint extracted from fingerphotos. Skin reflectance can be measured in a variety of ways, by capturing the RGB values of the skin, by capturing the melanin value, or capturing the erythema value of the skin. The melanin value represents the pigment of the skin and the erythema represents the redness of the skin. These methods differ depending on the hardware used to take the reading and can provide different insights into the resulting effect the skin reflectance may have.

\subsection{Organization of the Thesis}

The first chapter of the thesis elaborates on the information that acts as a basis for this work and provides an overview of the differences and effects the mentioned fingerprinting techniques have. Beyond the fingerprints, these sections also provide insight into the further effects that contactless fingerprints have when used in fingerprint matching due to the result of the contactless acquisition process. These sections also discuss alternative fingerprint matching techniques and how they relate and differ. The information in this section uses other published works to outline a basis for the research being presented.

The second chapter explores the underlying technologies used by both contact and contactless fingerprinting systems as well as fingerprint matching systems. These technologies are the building blocks used to create the commercial fingerprinting systems described in the introduction and backgrounds sections. The chapter is broken down into sensor hardware and fingerprint matching, with subsections that describe the relevant technologies.

The third chapter discusses the data used in the study and breaks down the relevant information and demographics of the data. The data is then broken down into what is present for each device and the amount that is present. Then, the matching experiments performed are presented in their execution and effect. These matching results make up the interoperability study of this work. In completing the experiments to perform the interoperability study, a baseline of contact-based fingerprint matching is presented for reference, as well as a baseline for unprocessed visual images that can be used to evaluate the results of the contactless devices presented. Along with the specific matching experiments, the studies performed are described focusing on the physiological factors that may have a noticeable and meaningful impact on matching results from one person to the next. 
The fourth- and fifth-chapters breakdown the outcomes of the described matching experiments. This breakdown provides the insight that can be taken away from these results and the quality of these results based on the distributions of the data. The conclusion then summarizes the outcomes of the general interoperability study and the experiments into the physiological aspects and whether they have a detrimental effect on the matching results for contactless fingerprints. After the conclusion is a final explanation of where and how this work can be continued in the future to further build on the results presented. 
Chapter 2:

Theory 


\subsection{Contact and Contactless Sensor Hardware}

\subsubsection{Frustrated Total Internal Reflection (FTIR)}

FTIR is the oldest and most commonly used method for acquisition of livescan fingerprints [1]. The contact-based fingerprint sensor using FTIR projects high contrast images across a Gradient Index of refraction (GRIN) lens array sensor [18]. The preferred capture method for this type of sensor involves sliding the finger across a transparent platen [18]. To view the fingerprint from the platen light is introduced from a flat or curved surface that collimates the light to form a sheet of light the width of the sensor [18] [19]. Once the light is introduced it can be directed using total internal reflection or reflected from a mirrored surface [18]. These methods of reflection allow the device being used to fit into a smaller form-factor [18]. The surfaces used for reflection of the light can be rough to allow the light to be diffused and cause more light to reach the fingerprint.

The use of an angle to view the transparent platen allows for a high contrast fingerprint image to be gathered using the aforementioned technique [18]. This image is focused with a GRIN lens array onto a linear sensor array [18]. Light in the system is directed to the top interior surface of the plated at a 45 degree or greater angle, where it is reflected by total internal reflection if there is no fingerprint in place [18]. When there are fingers present the light is not reflected and is absorbed providing bright areas for the valleys and dark areas for the ridges of the fingerprint [18] [19]. An example of this system is shown in Figure 2.1. 


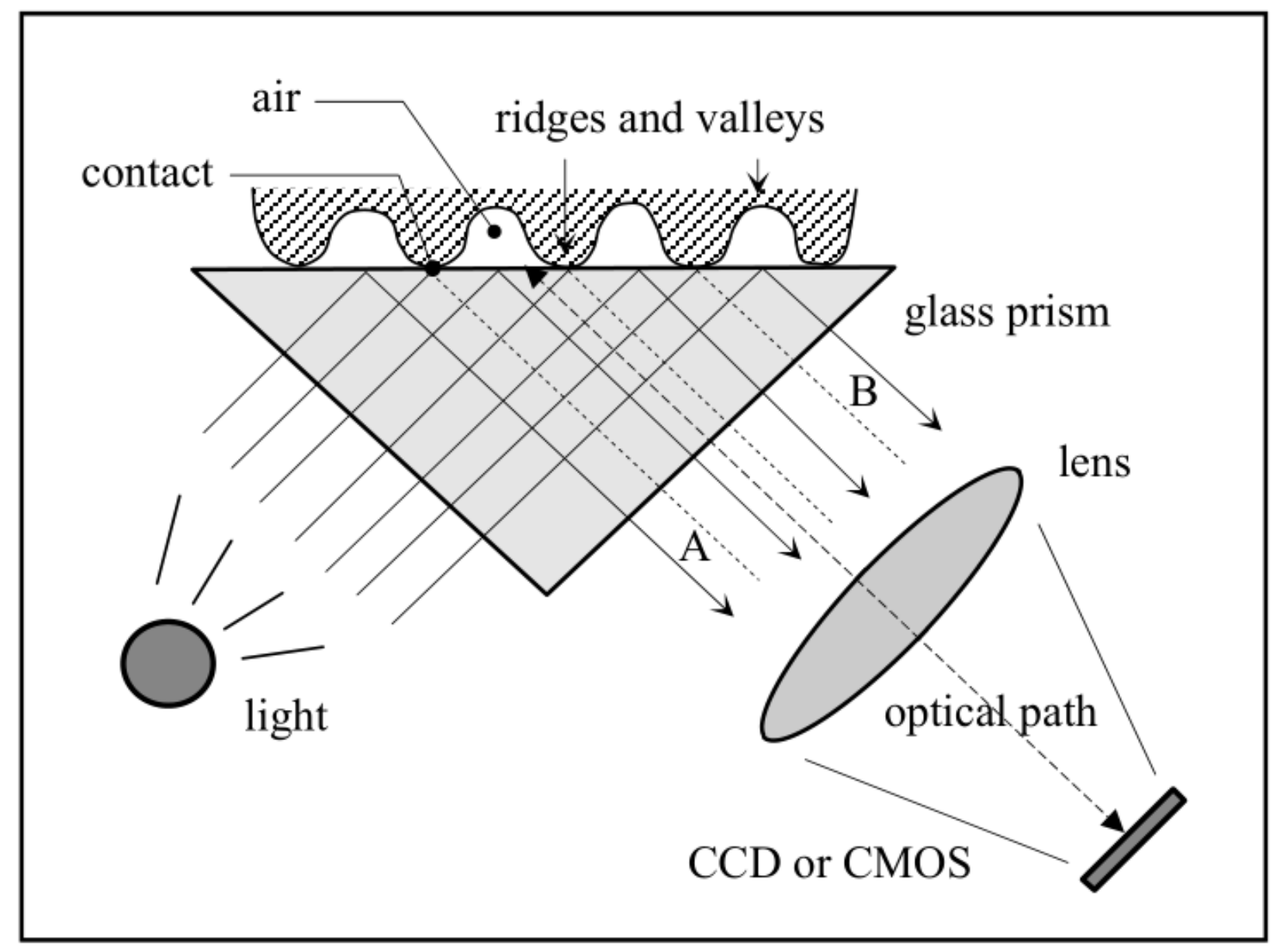

Figure 2.1: FTIR-based Fingerprint Sensor Operation [18].

\subsubsection{Structured Light Illumination}

Structured light illumination is a common technique used to captured fingerprints without requiring physical contact to the device. In a system using this technique fine grid structured lights illuminate the finger from many directions while cameras from multiple directions are used to capture the image [20]. These images are then processed to generate three dimensional or hybrid two dimensional images [20]. This process can capture all the information from the finger as well as the palm at the same time [20]. The use of multiple camera angles allows the resulting images to extract all of the detail from the finger without losing information that may not be present at all angles [20]. Structured light illumination uses at least one system for projecting the structured light pattern onto the fingers or hand in the capture area [21]. The system uses one or more cameras to capture images of the hand in the capture area [21]. The system then uses predetermined points in the backdrop of the capture area to combine the multiple images into a 
two-dimensional image that represents a traditional contact-based fingerprint [21]. An example of this three-dimensional mapping is shown in Figure 2.2.

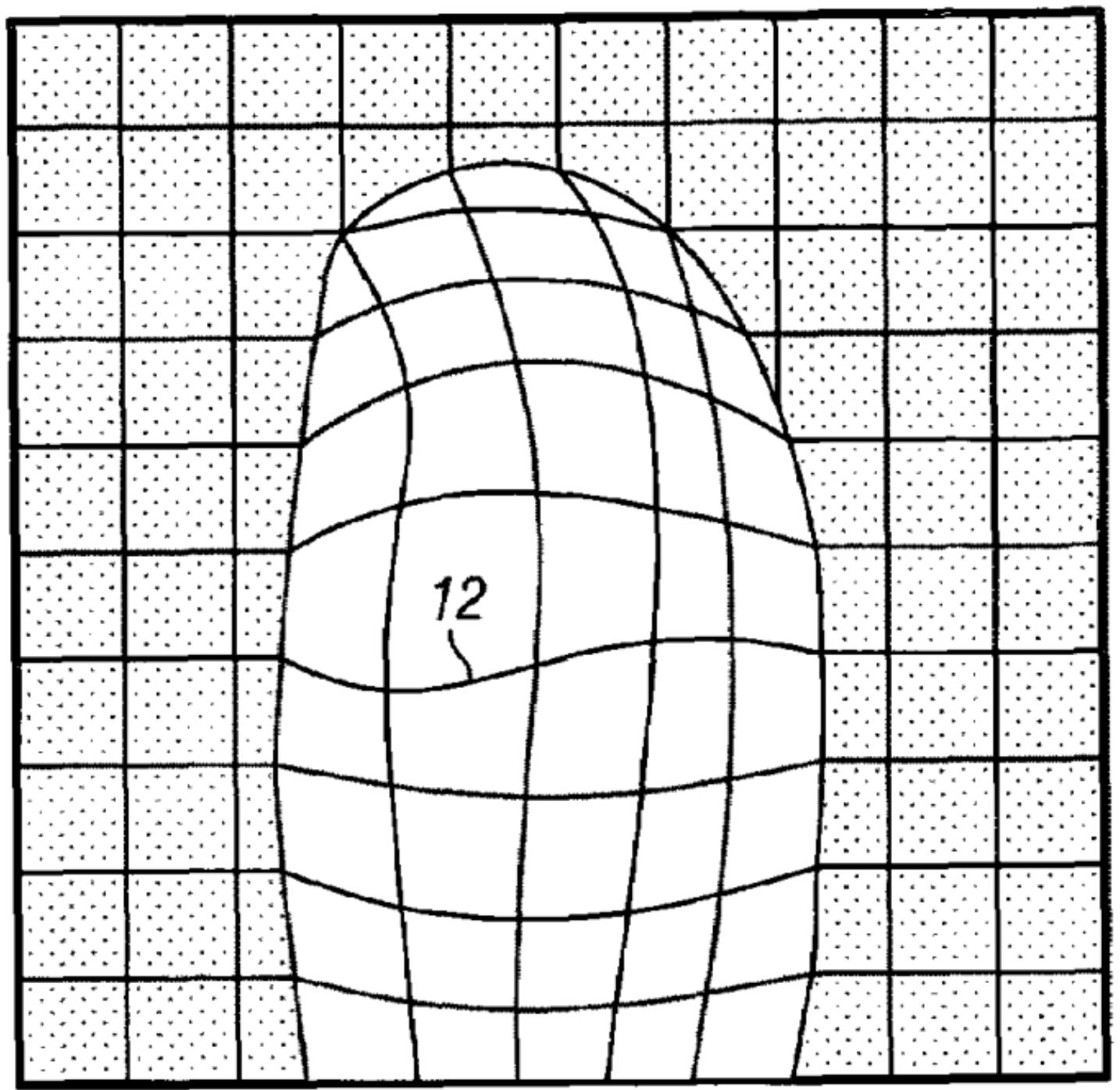

Figure 2.2: Example of Structured Light Illumination Applied to a Finger [20].

\subsubsection{Polarized Reflected Light}

Polarized reflected light is a method that allows for contactless capture of three-dimensional fingerprints using polarization to provide light from many angles [22]. The system used in this method involves a liquid crystal panel (LCP) and a birefringent element that are located between the finger and the capture sensor that polarizes the light passing from the finger to the capture sensor [23]. This process captures many polarized images from many rotation angles throughout 
the capture process [22]. The system can have polarization filters of a variety of angles typically between 0 and 90 degrees [23]. The images captured from all of the polarization angles of the LCP are combined to create a composite image [22]. In the process of creating the output image the many polarized images are used to calculate depth in three-dimensional space that can be used to generate the flattened two-dimensional image [22].

\subsection{Fingerprint Matching}

\subsubsection{Fingerprint Matching and Recognition}

Fingerprint matching is the process of quantifying the similarity of two fingerprint images [24]. Fingerprint matching is a common technique used by law enforcement agencies to identify persons based on fingerprints left at the scene of a crime, as well as, to identify offenders that have had their fingerprints recorded [25]. Fingerprint recognition systems cover a broader scope of uses including both verification and identification based on the use case. Verification covers a one-to-one approach of comparing a fingerprint with another fingerprint that has been previously enrolled [25]. Identification covers the process of comparing one fingerprint against a gallery of fingerprints in a one-to-many configuration of comparison [25]. The comparison of fingerprints based on similarity can be done using a variety of techniques, but the most common method is minutiae based fingerprint matching [8] [25] [26] [27] [28].

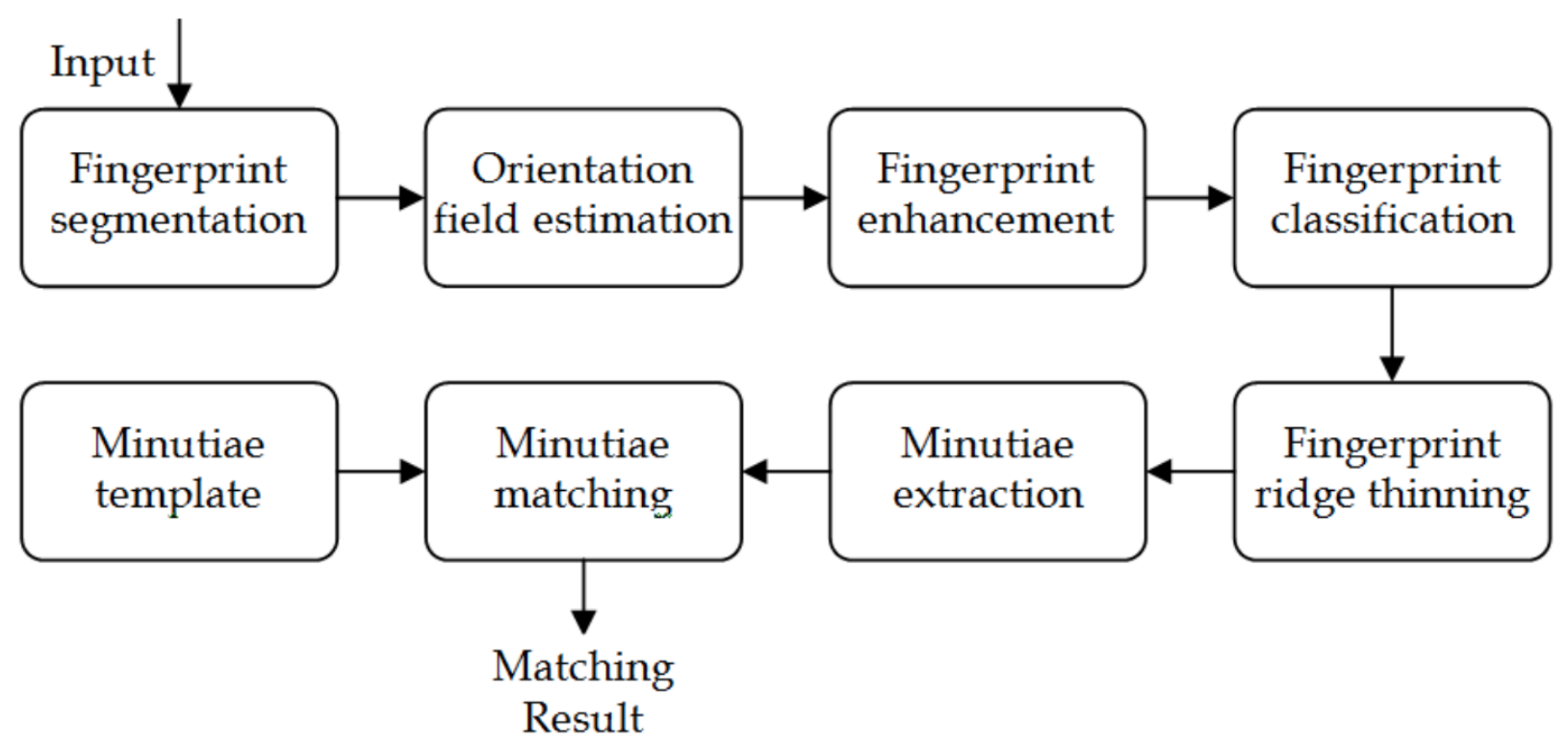

Figure 2.3: General Block Diagram for a Fingerprint Identification System [28]. 
Fingerprint matching consists of three independent systems: the fingerprint sensor or capture device, feature extraction, and the matcher. The fingerprint sensor is the device that captures the fingerprint used in the rest of the system [8]. Feature extraction is the method of processing the fingerprints to extract the features used by the matcher for comparison [8]. The features that are extracted are the position and orientation of minutiae, the unique ridge and valley combinations across the fingerprint [8]. The minutiae extracted from the fingerprint can be classified into three levels [25]. Level 1 features are broad details such as singular points, friction ridge flow, and pattern type [25]. Level 2 features are the ridge endings and bifurcations [25]. Level 3 features include all of the minute details of the ridges including ridge path deviations, ridge width, shape, pores, etc. [25].

The matcher is the final major component of the matching system that compares the input fingerprints against the stored gallery of templates [8]. The matcher compares the minutiae from the input and gallery images and produces a similarity or match score between the two [8]. Using this system, the match scores between fingerprints of the same finger trend higher and for different fingers lower [25]. The system for matching has its own challenges such as, the variation in fingerprint images from the same finger across different capture conditions and devices, as well as the potential similarity of fingerprints captured from different fingers [25].

Minutiae based matching uses local minutiae structures to find a coarse alignment for the fingerprints to provide proper global alignment to assess the fingerprints' similarity [25]. A breakdown of the common steps in a system as described from [25] is shown in Figure 2.4. The system starts by finding the pairwise similarity using minutiae that are static regardless of the orientation [25] [28]. These minutiae pairs are then used to align the overall fingerprint for full similarity consideration [25]. The minutiae between the two prints are then considered to find the minutiae pairs that are considered mated between both prints [25]. Finally, the similarity score is computed between the two fingerprints by considering the percentage of matching minutiae and the ridge consistency between the mated minutiae [25]. 


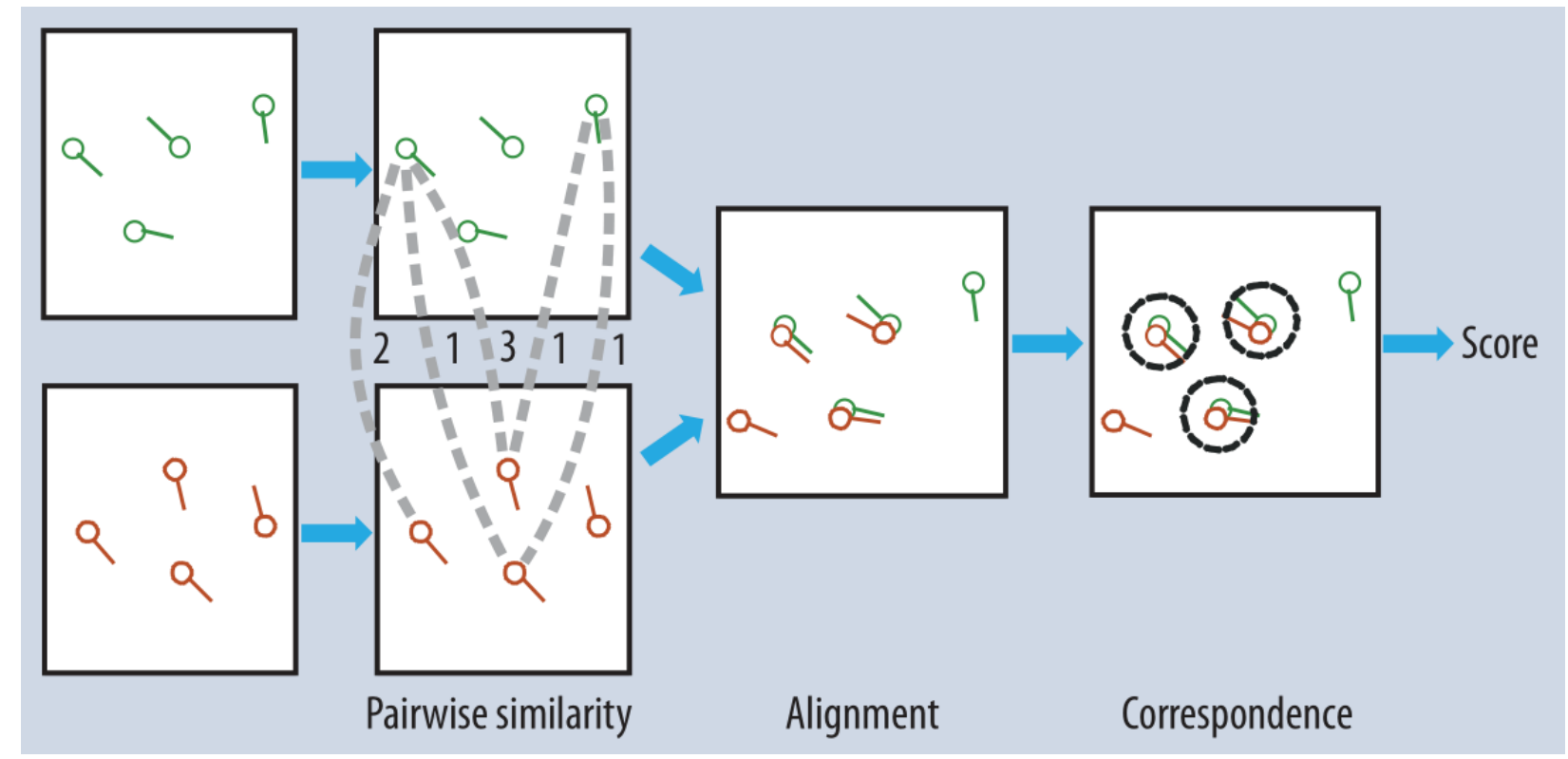

Figure 2.4: Typical Minutiae Matching Algorithm [25].

\subsubsection{Matching Errors}

Matchers can exhibit two main errors when matching fingerprints, false matches and false nonmatches [8] [25]. A false match is the result of the matcher producing a high similarity between two fingerprints from different fingers [8] [25]. A false non-match is the result of the matcher producing a low similarity between two fingerprints captured from the same finger [8] [25]. The false match can be referred to as a false accept and the false non-match can be referred to as a false reject when discussed [8] [25].

\subsubsection{Receiver Operating Characteristic Curves}

A receiver operating characteristic (ROC) curve is a plot that compares the true positive rate against the false positive rate across a variety of match score thresholds [29]. The ROC curve serves as a method for evaluating the matching or classification performance of a system [30]. The closer the curve follows the left and top borders of the plot, the higher the accuracy of the overall system [29]. The slope of a tangent line at any given point or threshold in the plot provides the likelihood ratio for that point [29]. An example ROC curve can be seen in Figure 2.5 . 


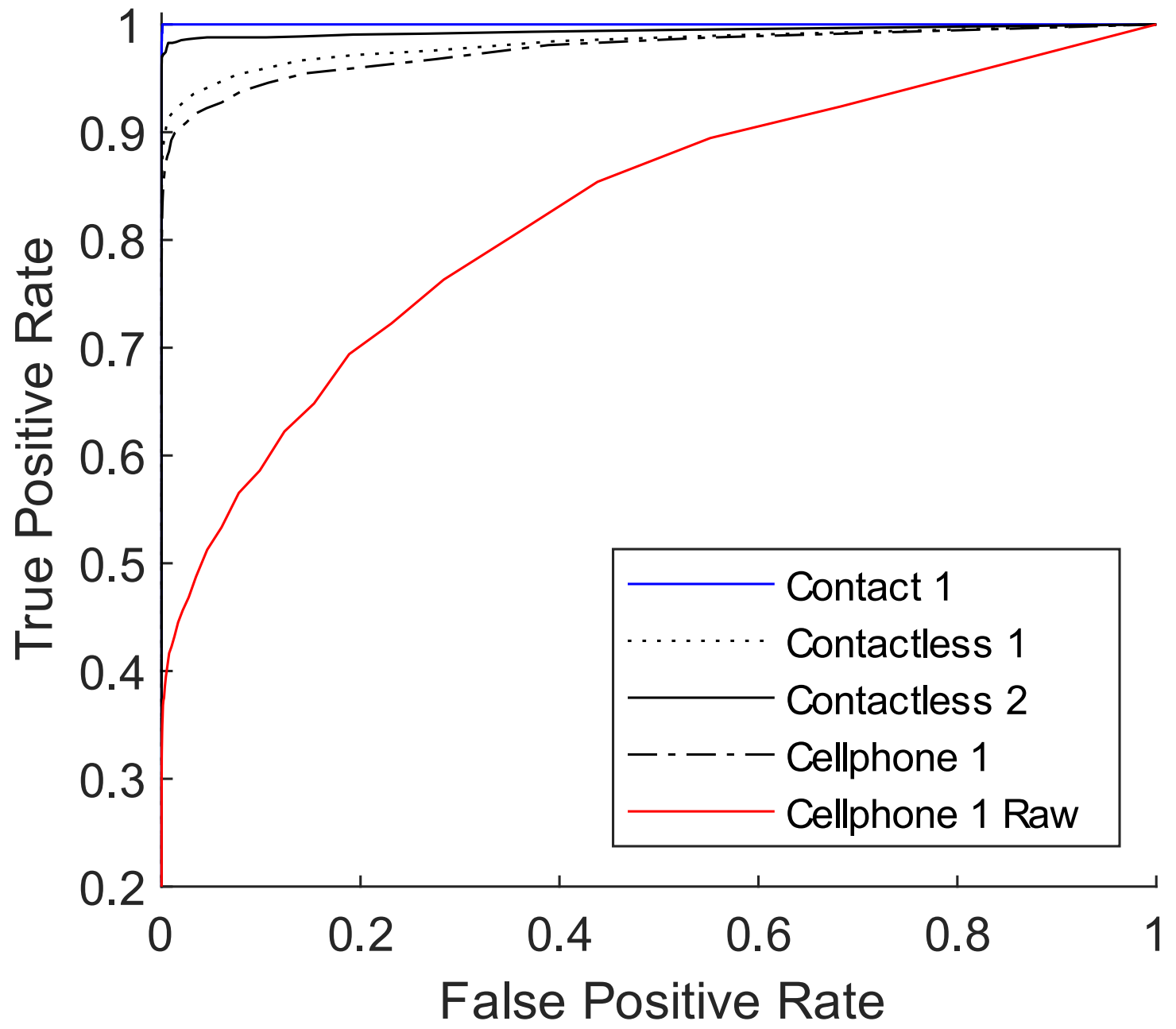

Figure 2.5: Example ROC Curve.

\subsubsection{NIST Fingerprint Image Quality (NFIQ)}

NIST Fingerprint Image Quality or NIST is an algorithm designed to assess and produce a quality value for fingerprint images [31] [32]. NFIQ was originally developed in 2004 then expanded and improved in 2014 with the release of NFIQ 2, and was created with goal of providing an assessment for the quality of recorded fingerprints and directly predict how they will perform for matching [31] [32]. The algorithm provides a score between 1 and 5, with 1 being the highest quality and 5 being the lowest [31] [32]. 


\section{Chapter 3: \\ Dataset Details and Matching Experiments}




\subsection{Data Collection}

The data used in the experiments described below were collected from an on-site data collection performed in the Biometrics Collections Lab under WVU IRB Protocol \# 2001870127 collection. The data collection was completed using six devices employed at five stations and during the registration process. After participants arrived at the lab, they proceeded to the consent and registration station where they filled out the informed consent form, provided basic demographic information (age, gender, ethnicity, etc.), and were assigned a random seven-digit identification number that was to be included in all of the filenames of the fingerprint image samples they provided. Here, they also had their palm and back of their hand scanned with a skin colorimetry device. From there, the participant would start at the hand geometry station, where images of the front and backs of their hands were collected using a Canon 5DSR DSLR camera. Then, in order, the following devices were used to capture fingerprints: a Gemalto contactless device, a Crossmatch (Now HID) contact-based device, and a Morpho contactless device. Finally, an iPhone app developed by Veridium was used to collect mobile contactless fingerprints with the built-in camera. An example diagram of the collection workflow is shown in Figure 3.1.1, as well as an actual photo of the setup shown in Figure 3.1.2.

\section{Operator}

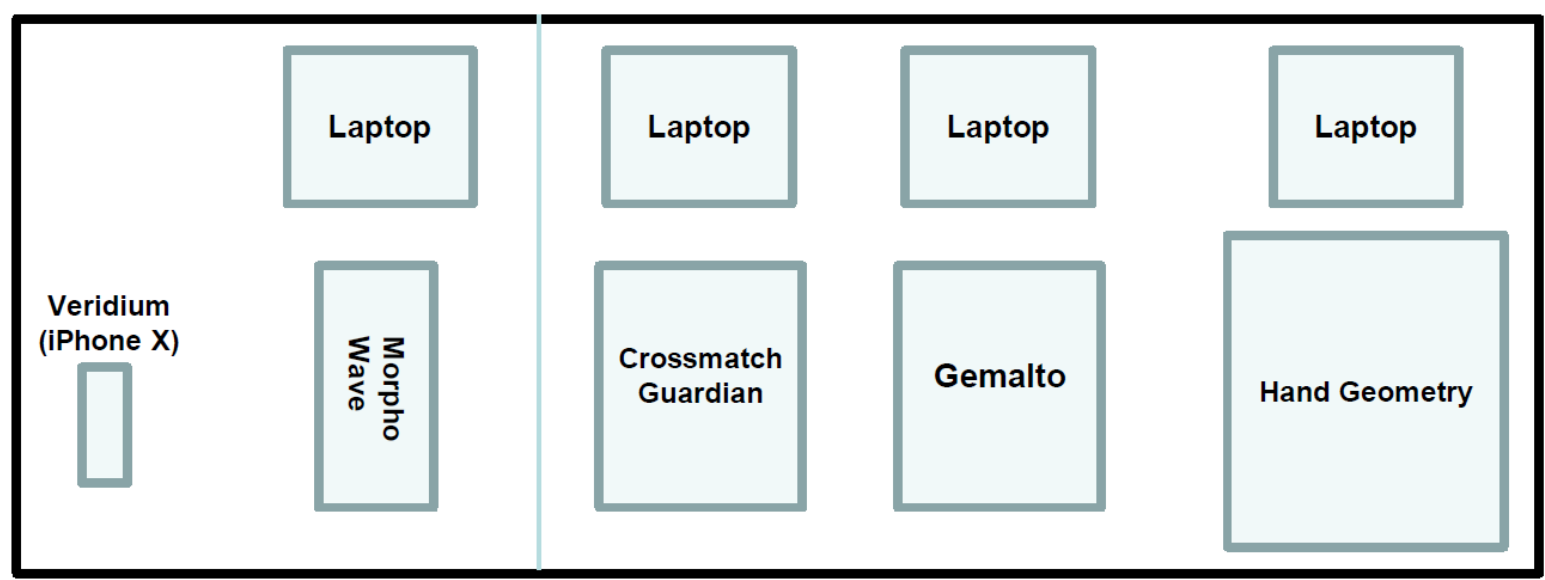

Participant

Figure 3.1.1: Data Collection Layout. 


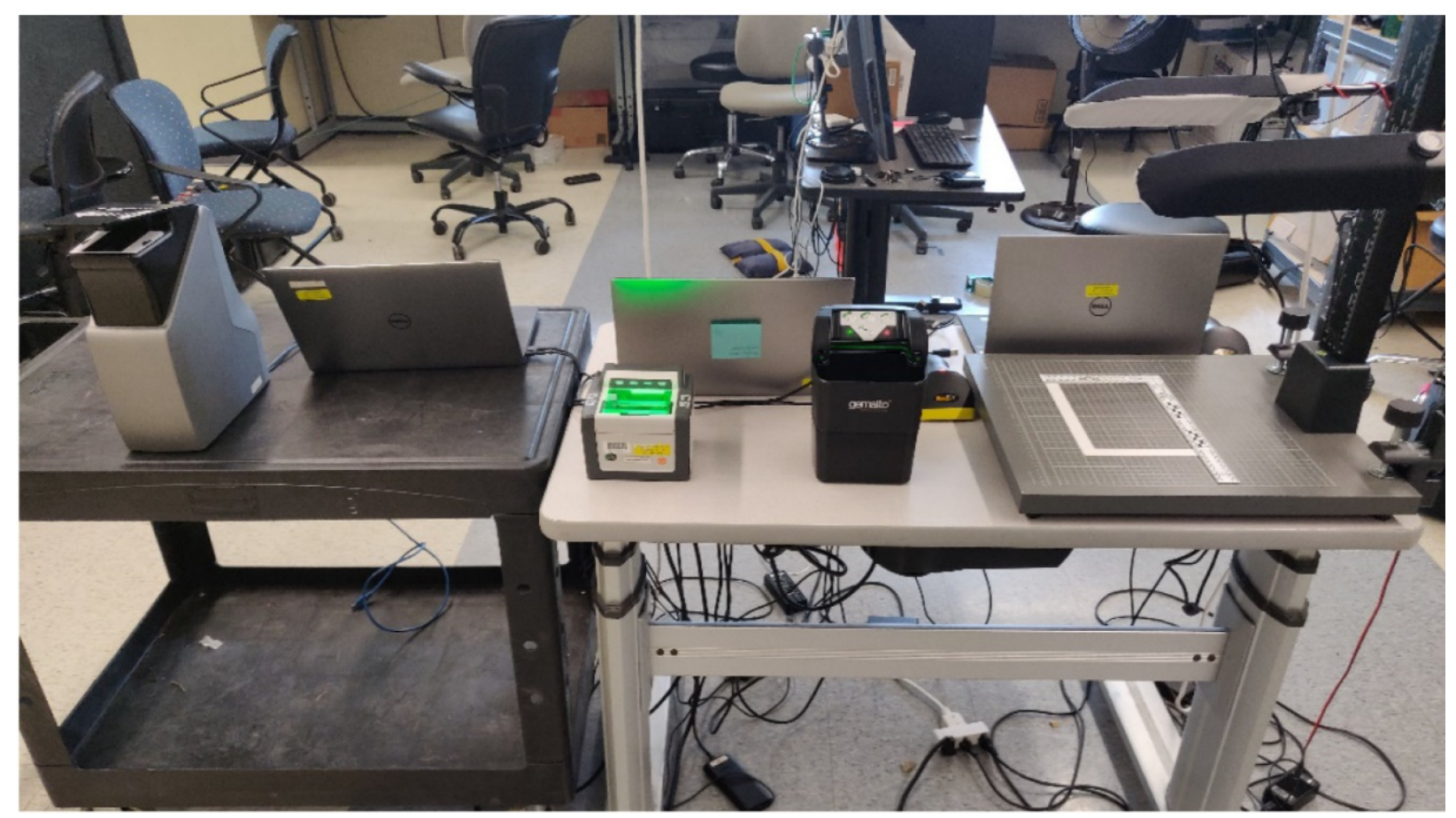

Figure 3.1.2: Data Collection in Progress Photo.

\subsection{Dataset Details}

The fingerprints used in these experiments were collected from 215 individuals who each provided fingerprint data across multiple commercial fingerprint capture devices. A summary of the number of images from each sensor is provided in Table 3.2.1. Some devices captured images across multiple sessions, with others only capturing one session. The total number of fingerprint images used in matching experiments was 1,165, consisting of fingerprints from the index, middle, ring, and little fingers only. Thumbs were excluded from the matching analyses because not every device captured thumbprints. The dataset also contains finger size data collected from hand geometry images and skin reflectance data measured using the Cortex Technology DSM III sensor [33]. The finger size data was calculated from the hand geometry images by setting the scale based on the $1000 \mathrm{~mm}$ pixel values from the ruler in the image, then by selecting the edges of the finger to convert the pixel values into millimeters for comparison. An example of this is shown in Figure 3.2.1. The skin reflectance data provides CIEL*a*b* RGB data and a measure of melanin and erythema in the skin [33]. Three breakdowns of participant demographics are shown in Figures 3.2.2-3.2.4. 


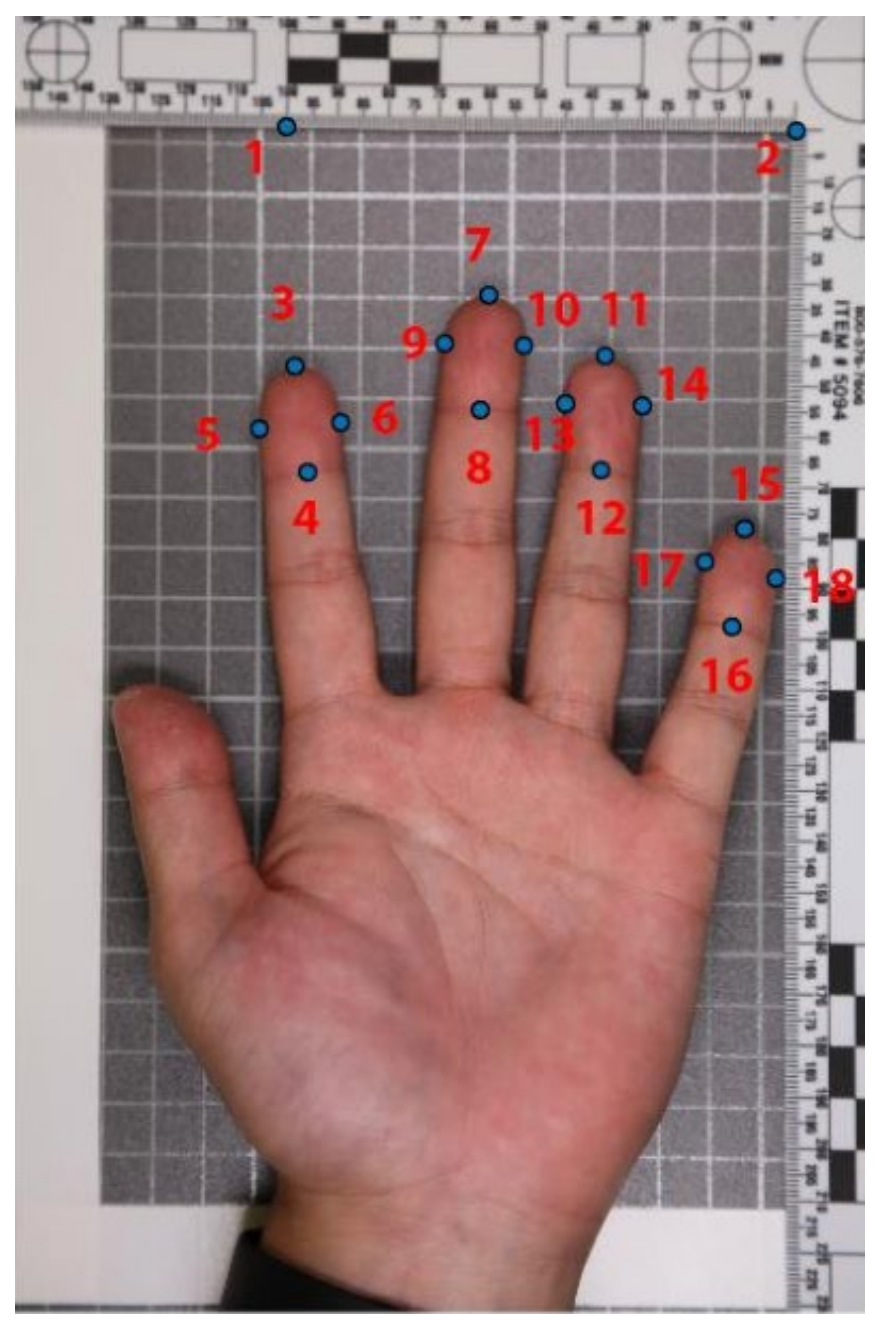

Figure 3.2.1: Finger Measurement Example.

Table 3.2.1: Dataset Description.

\begin{tabular}{|c|c|c|c|c|}
\hline Device & \multirow{2}{*}{ Image Type } & No. of Samples & No of & Tessions \\
\hline Contact 1 & slaps \& rolls & 2 slaps 2 thumbs 10 rolls & 1 & 4300 \\
\hline Contactless 1 & slaps & 2 slaps 2 thumbs & 1 & 2150 \\
\hline Contactless 2 & slaps & 2 slaps 2 thumbs & 2 & 4300 \\
\hline Cellphone 1 & slaps & 2 slaps & 3 & 5160 \\
\hline
\end{tabular}




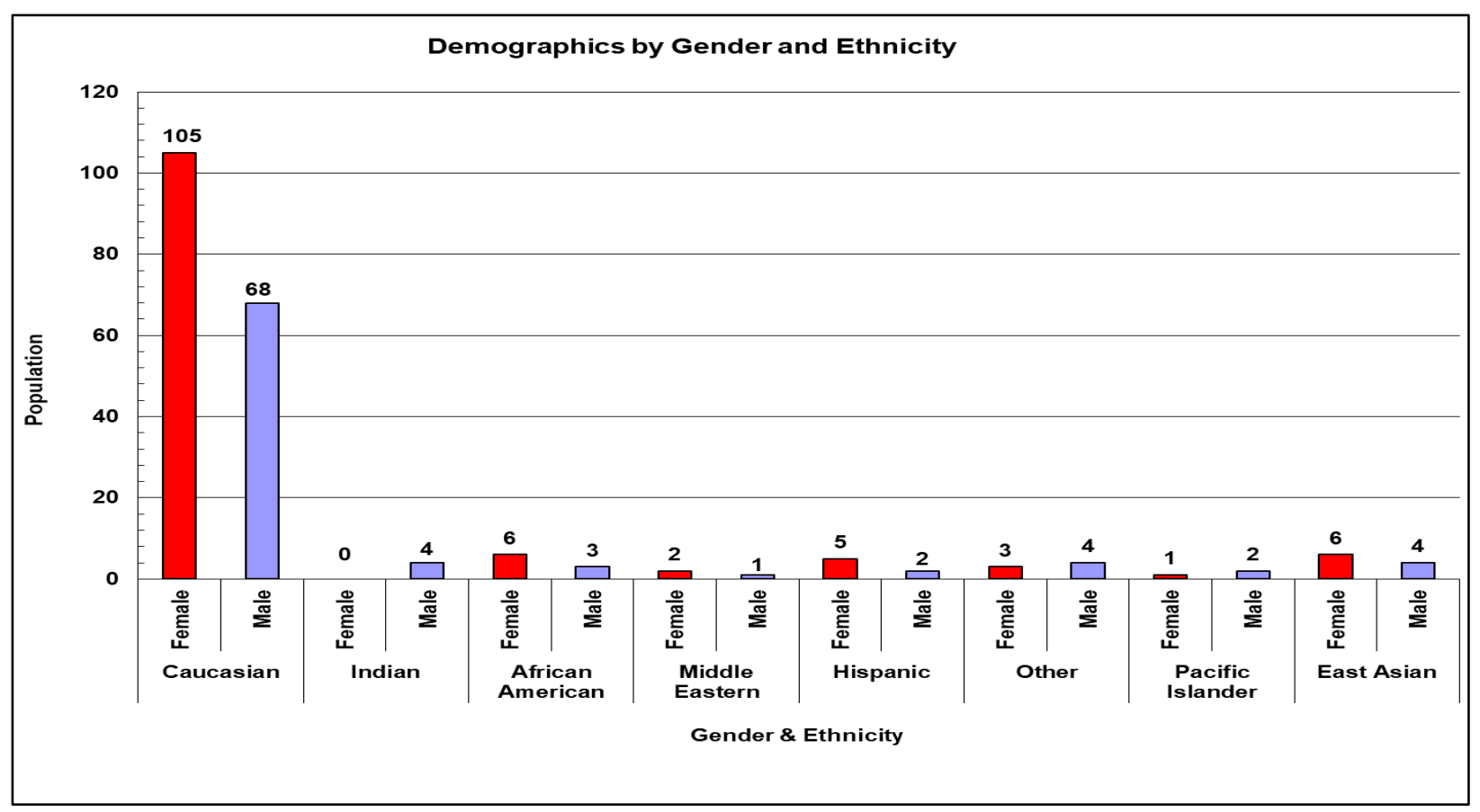

Figure 3.2.2: Participant Demographics by Gender and Ethnicity.

\section{Participants by Ethnicity Group (\%)}

\begin{tabular}{|c|}
\hline CAUCASIAN \\
\hline EAST ASIAN \\
\hline = INDIAN \\
\hline $\begin{array}{l}\text { AFRICAN } \\
\text { AMERICAN }\end{array}$ \\
\hline $\begin{array}{l}\text { MIDDLE } \\
\text { EASTERN }\end{array}$ \\
\hline = HISPANIC \\
\hline $\begin{array}{l}\text { PACIFIC } \\
\text { ISLANDER }\end{array}$ \\
\hline " OTHER \\
\hline
\end{tabular}

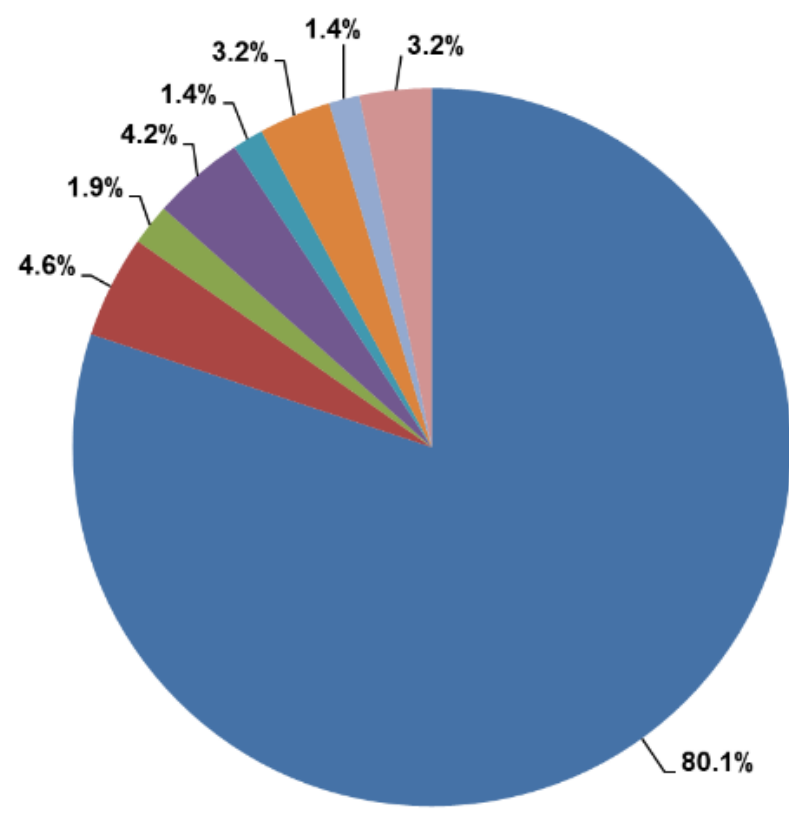

Figure 3.2.3: Participant by Ethnicity Group. 


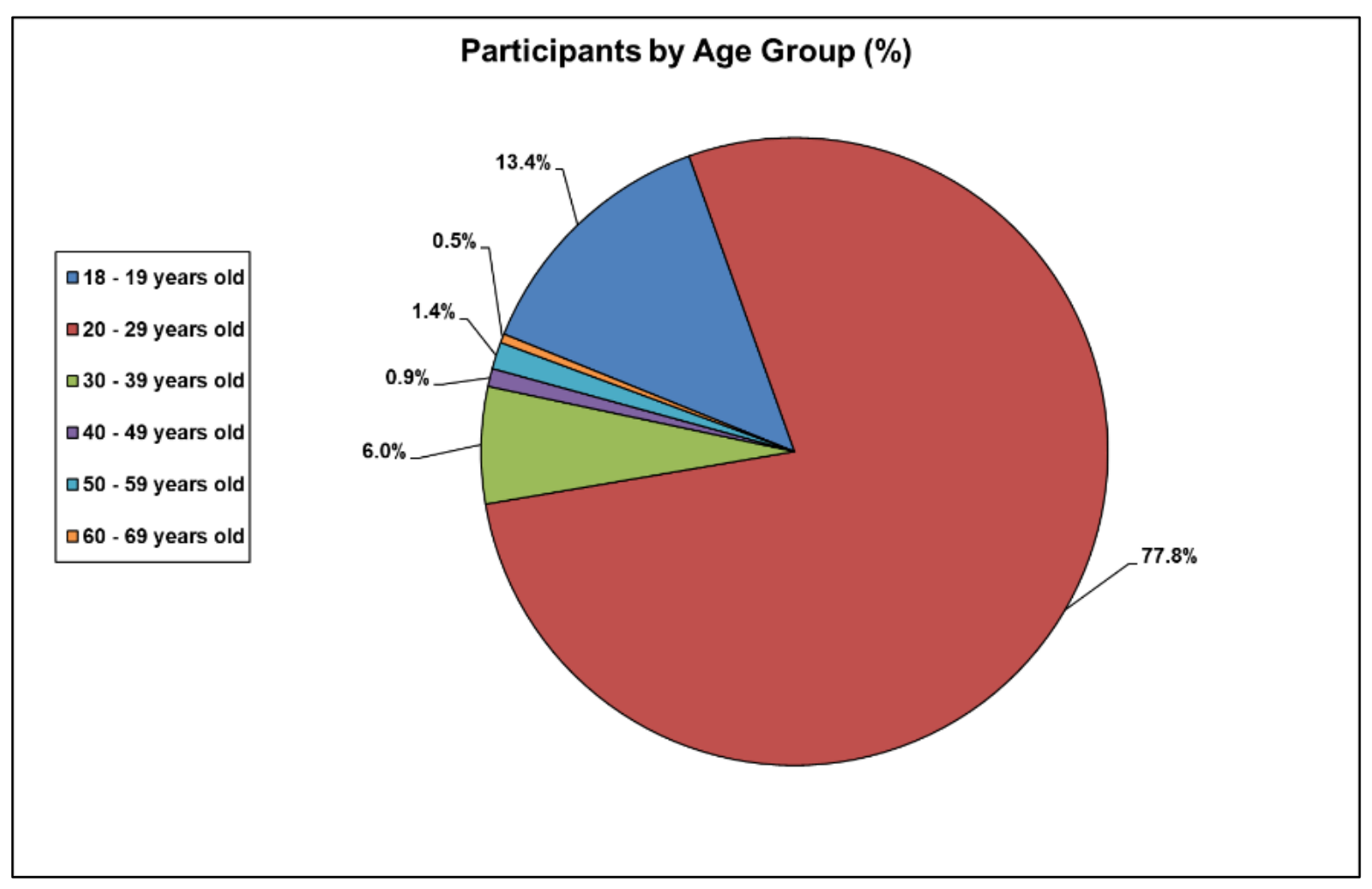

Figure 3.2.4: Participants by Age Group.

\subsection{Matching Experiments}

Before matching, preprocessing was performed on the raw versions of the cellphone-based fingerphotos, a step-by-step example of this preprocessing is shown in Figure 3.3.1. The photos were converted to grayscale, histogram equalization was applied, and they were inverted so the ridges are shown as the dark regions of the fingerprint to match traditional fingerprinting techniques. These processed photos, referred to as Cellphone-1-Raw, were matched to provide a comparison of the fingerprint processing done by the COTS application in Cellphone- 1 . Along with the raw photos, the cellphone-based application provided binarized generated prints from the photos that were also used in matching (i.e., Cellphone-1 images). 


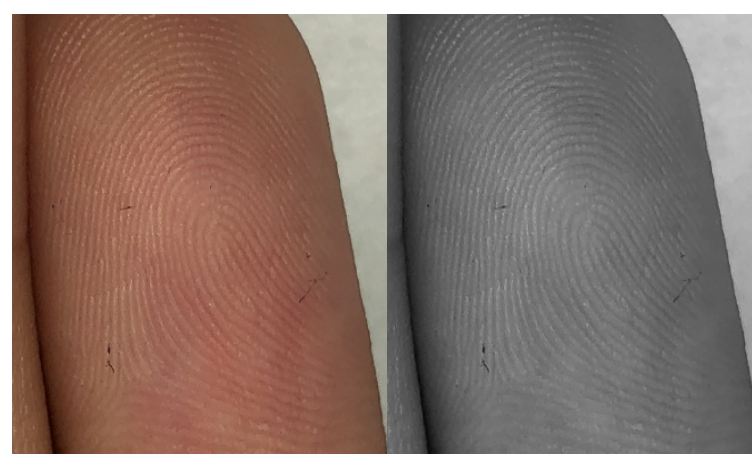

(a)

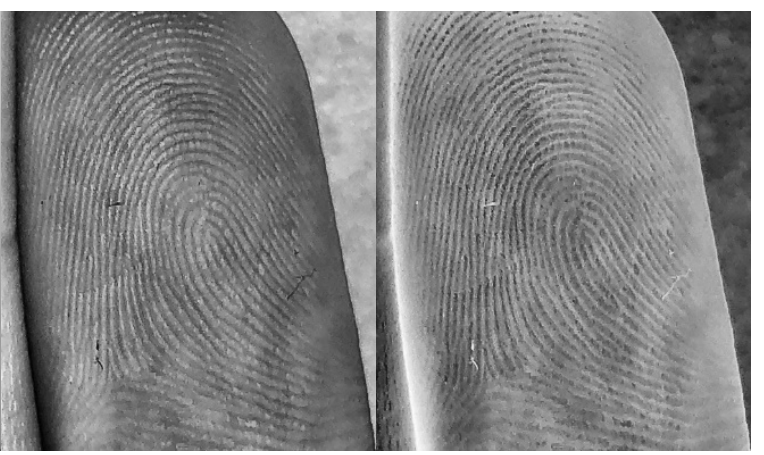

(c)

(d)

Figure 3.3.1: Step-by-Step Preprocessing for Cellphone-Based Fingerphotos Showing (a) Original (b) Grayscale (c) Adaptive Histogram Equalization (d) Inverted.

Using this dataset, matching experiments were performed on two commercial black-box matchers and one open-source matcher with the segmented slap fingerprints from Contact-1 as the gallery for all matches. The two commercial black box matchers and the open-source matcher are referred to as Matcher-1, Matcher-2, and Matcher-3, respectively. All matches were performed in a one-to-many configuration so that scores were generated for all probes versus all gallery images. The threshold for all matchers was set to 0 to allow all match results to be extracted. As a baseline for the match scores, the rolled fingerprint data that was collected with Contact-1 was matched against the gallery of segmented slaps used for all other matches. Using the results of these matching experiments, receiver operating characteristic (ROC) curves were generated and the area under the curve (AUC) was calculated for each. The AUC provides general insight into the matching performance in line with the interoperability analysis being performed. This was followed by a statistical analysis of the matching results and a statistical correlation of the finger size and skin reflectance data with the matching results.

The analysis of the impact of finger size on contactless fingerprint match performance was performed using the width of the middle finger of the right hand of all individuals. Using this measurement, the finger sizes were split into equal-sized groups and the mated match scores were sorted into these groups to produce a distribution for analysis. The mated match scores were scores obtained by comparing two fingerprint images collected from the same finger. The analysis for the skin reflectance data involved splitting the data into three equal-sized ranges of melanin value using the melanin value provided by the DSM III. From there, a distribution was generated using the mated match scores to evaluate any effect caused by the amount of melanin on the resulting scores. 


\section{Chapter 4: Matching Results}


The results of the matching experiments are provided in Figures. 4.1-4.31, along with a discussion of the results.

The results shown in Figures 4.1-4.3 are ROC curves for the contactless devices compared against Contact-1 as well as the baseline match using Contact-1. Along with the contact baseline, there is a 'worst-case' baseline determined using the preprocessed raw images from Cellphone-1 to show a difference in performance when using the binarized images produced by the cellphone app. The results show a clear distinction in match performance between the three devices that is consistent for the three matchers used.

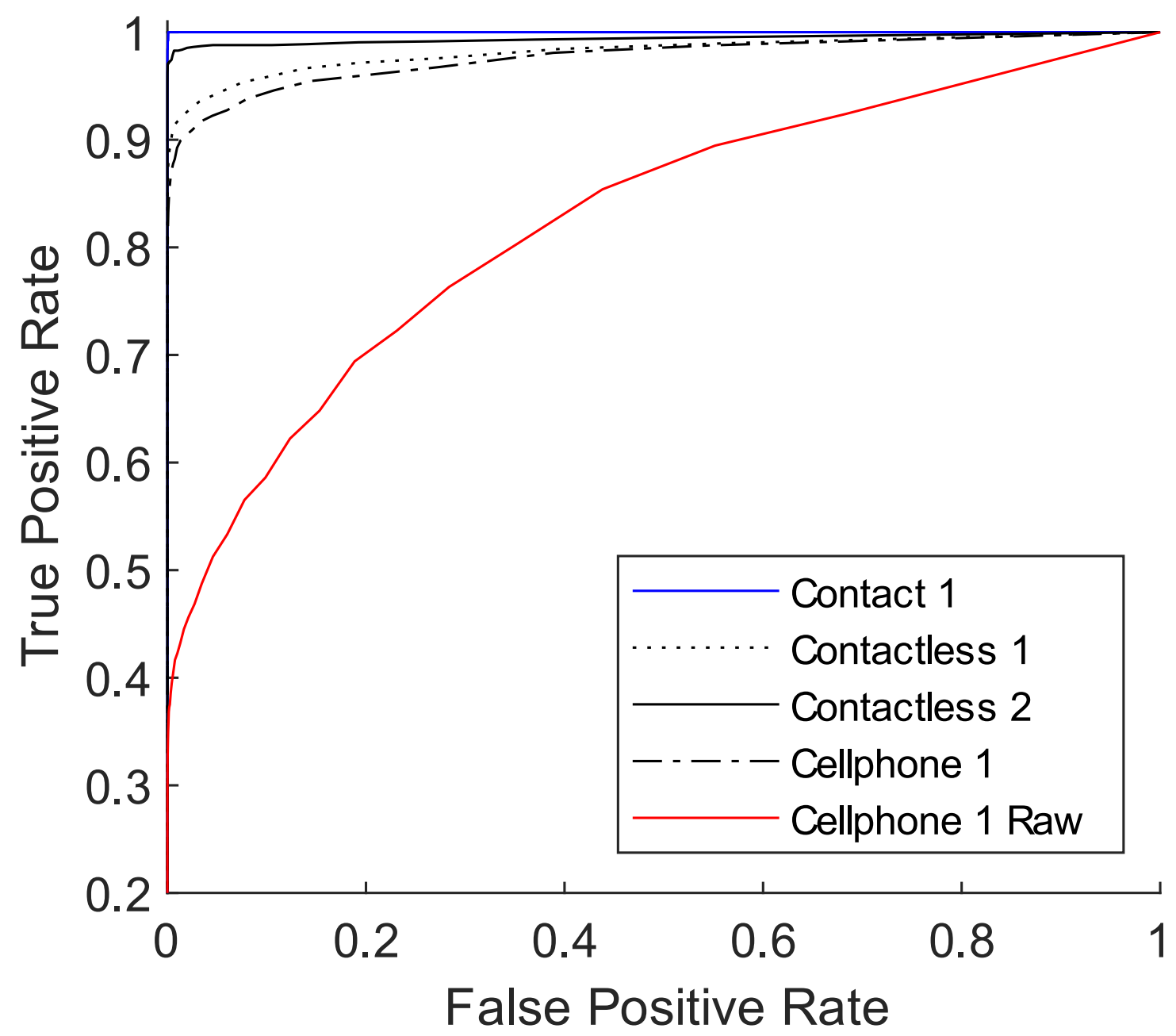

Figure 4.1: Receiver Operating Characteristic for contact and contactless fingerprint devices against Contact-1 using Matcher-1. 


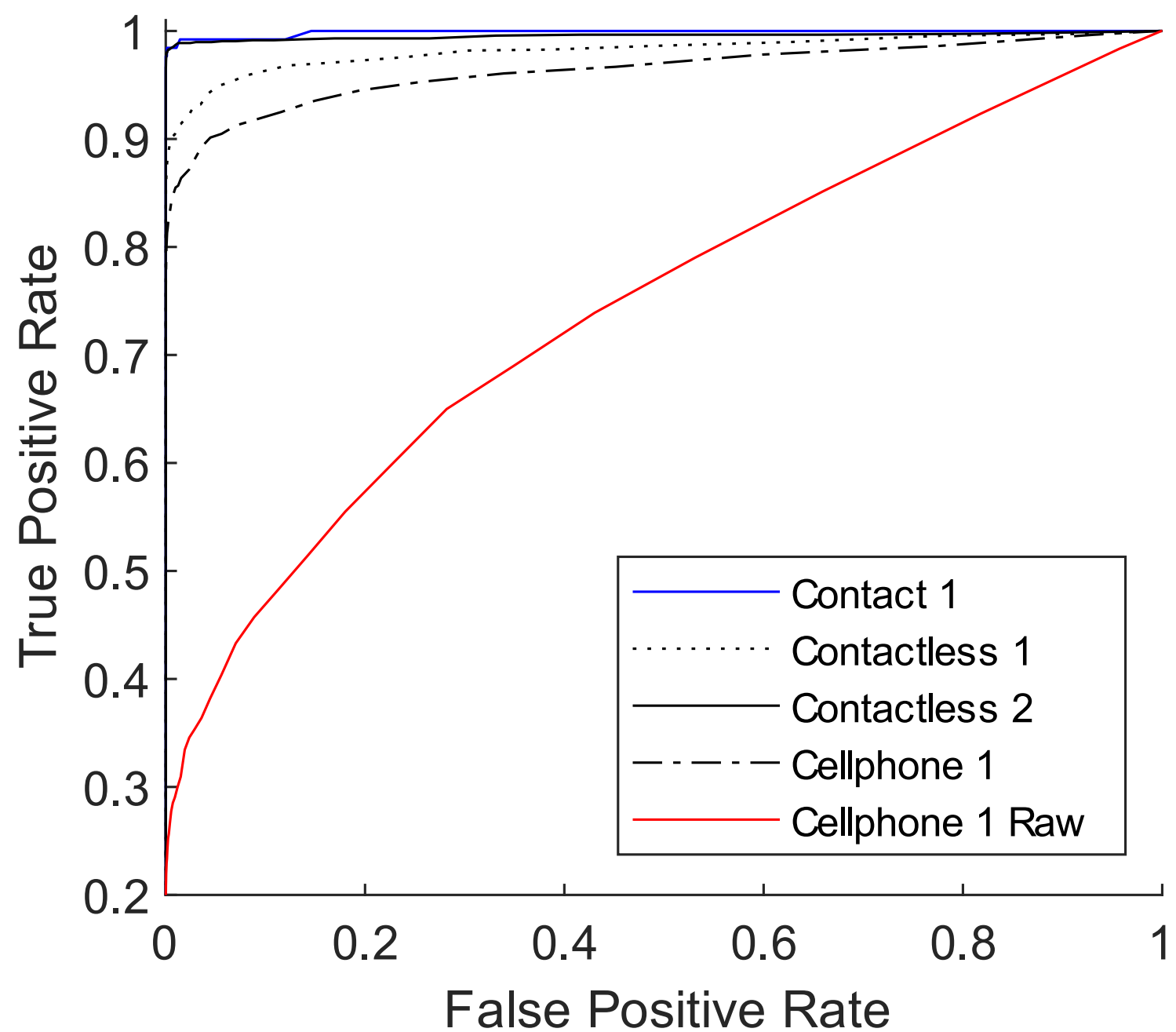

Figure 4.2: Receiver Operating Characteristic for contact and contactless fingerprint devices against Contact-1 using Matcher-2. 


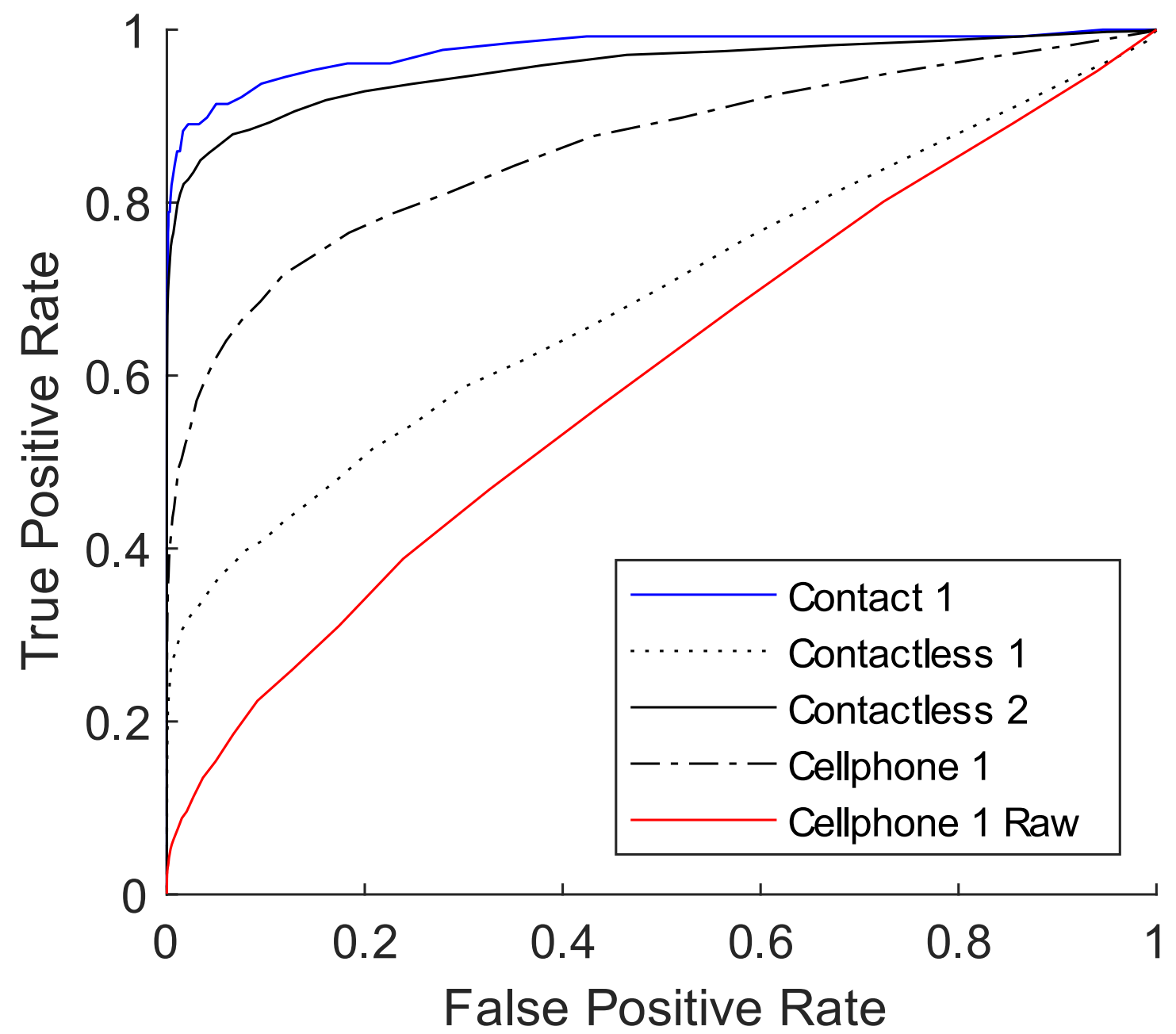

Figure 4.3: Receiver Operating Characteristic for contact and contactless fingerprint devices against Contact-1 using Matcher-3.

As is shown by the area under the curve (AUC) calculated from the ROC curves, shown in Table 4.1, Cellphone-1 exhibited the worst matching performance out of the three contactless sensors for the first two matchers, but only by a small margin below Contactless-1. Of the contactless images used in Matcher-3, Contactless-1 performed the worst with an AUC of only 0.6897. Based on these AUC values Contactless-2 consistently showed the best performance of the contactless devices and just fell short of the results of the contact baseline. As expected, Cellphone-1-Raw performed the worst across the board. This outcome was expected given that all of the fingerprint images provided by the other devices were prepared in a way as to focus on the relevant parts of the fingerprint, which was not the case for the raw cellphone images. 
Table 4.1: AUC of ROC Curves.

\begin{tabular}{|c|c|c|c|}
\hline Device & Matcher-1 & Matcher-2 & Matcher-3 \\
\hline Contact-1 & 1.0000 & 0.9989 & 0.9765 \\
\hline Contactless-1 & 0.9818 & 0.9820 & 0.6897 \\
\hline Contactless-2 & 0.9940 & 0.9955 & 0.9551 \\
\hline Cellphone-1 & 0.9764 & 0.9635 & 0.8606 \\
\hline Cellphone-1-Raw & 0.8252 & 0.7422 & 0.5964 \\
\hline
\end{tabular}

Based on this data the match results using Matcher-3 exhibited lower accuracy when compared to the other matchers. All devices performed similarly in all experiments, except for Contactless1, which had a much lower matching accuracy on Matcher-3, below the performance of the processed images from Cellphone-1. It should be noted that all matchers were used in an 'out of the box' configuration with no optimization for minutiae detection in contactless prints to keep the matching results fair.

Figure 4.4 shows the box plot for the NFIQ scores for all devices as well. These scores range from 1 to 5 with 1 being the highest quality fingerprints for matching and 5 being the worst. As expected, both the plain impressions and rolled prints from Contact-1 show high NFIQ scores trending toward the best value of 1 . Based on the ROC plots shown combined with these results the performance of Contactless- 1 and Cellphone- 1 center around a value of 2 but have a wide variety of scores across the 1-5 scale used. This contrasts with Contactless-2 that centers around a score of 3 but the range of scores is much smaller. As to be expected, Cellphone-1-Raw tends toward higher scores indicating a loss in quality for matching performance. Based on these results specifically comparing Contactless-1 and Contactless-2, both their NFIQ scores and ROC plot performance, it can be inferred that a slightly worse but more consistent NFIQ score may be more conducive to increased matching performance. Of note as well, NFIQ was designed with contact-based fingerprints as the standard and that is shown in the performance of the scores of the contact-based images used. 


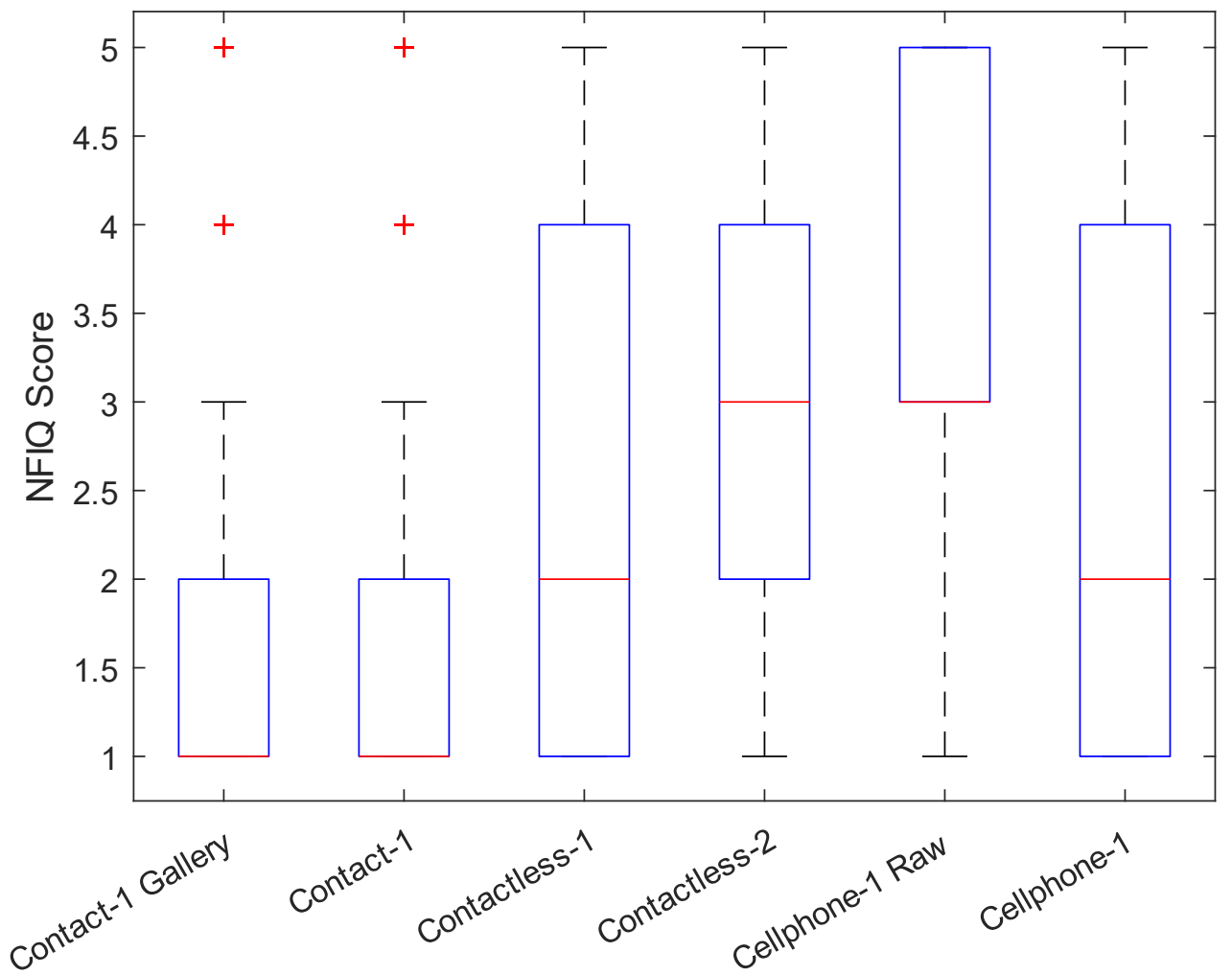

Figure 4.4: NFIQ Score Distribution for All Devices.

The results shown in Figure 4.5-4.7 are a comparison of the mated match scores for each of the devices using a specific matcher. In agreement with the ROC curves, the match scores of the two contactless devices trend higher than Cellphone-1, with Contactless-2 achieving the highest match scores consistently. As expected, Contact-1 trends higher overall for all matchers, while all of the contactless devices fail to reach the high end match scores that are present in the contact-based fingerprints. 


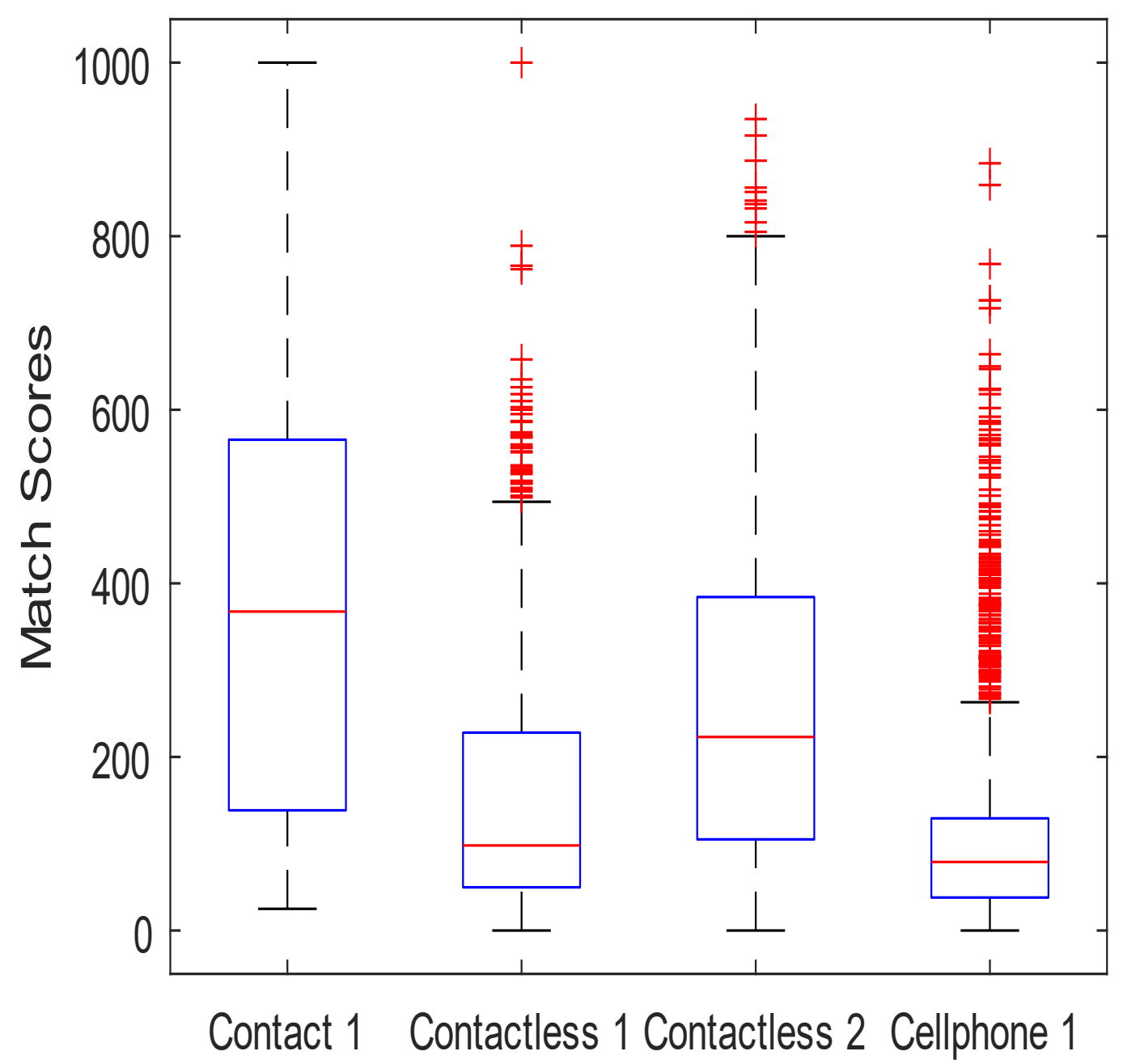

Figure 4.5: Comparison of the distribution of mated match scores for each device using Matcher-1. 


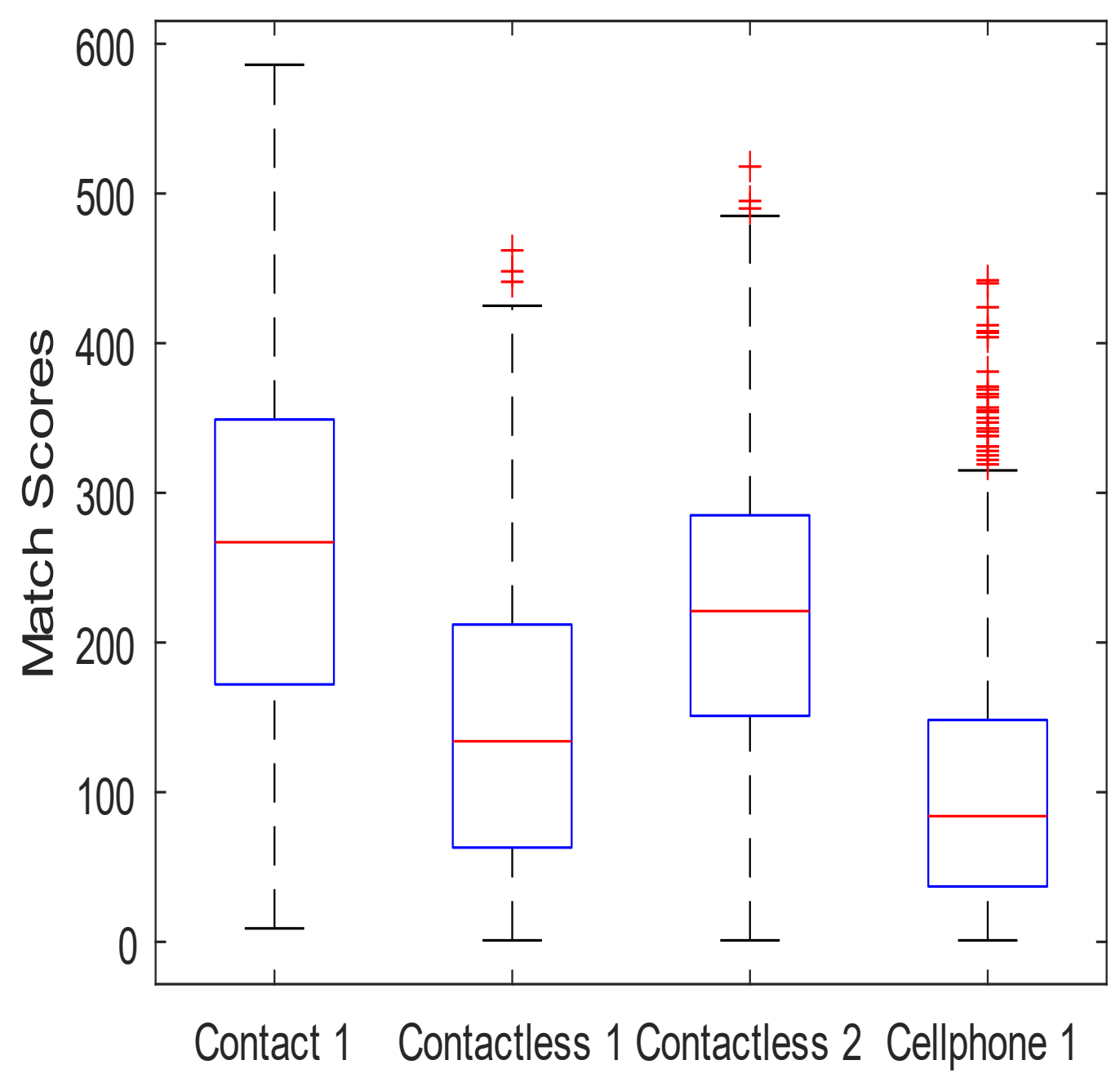

Figure 4.6: Comparison of the distribution of mated match scores for each device using Matcher-2. 


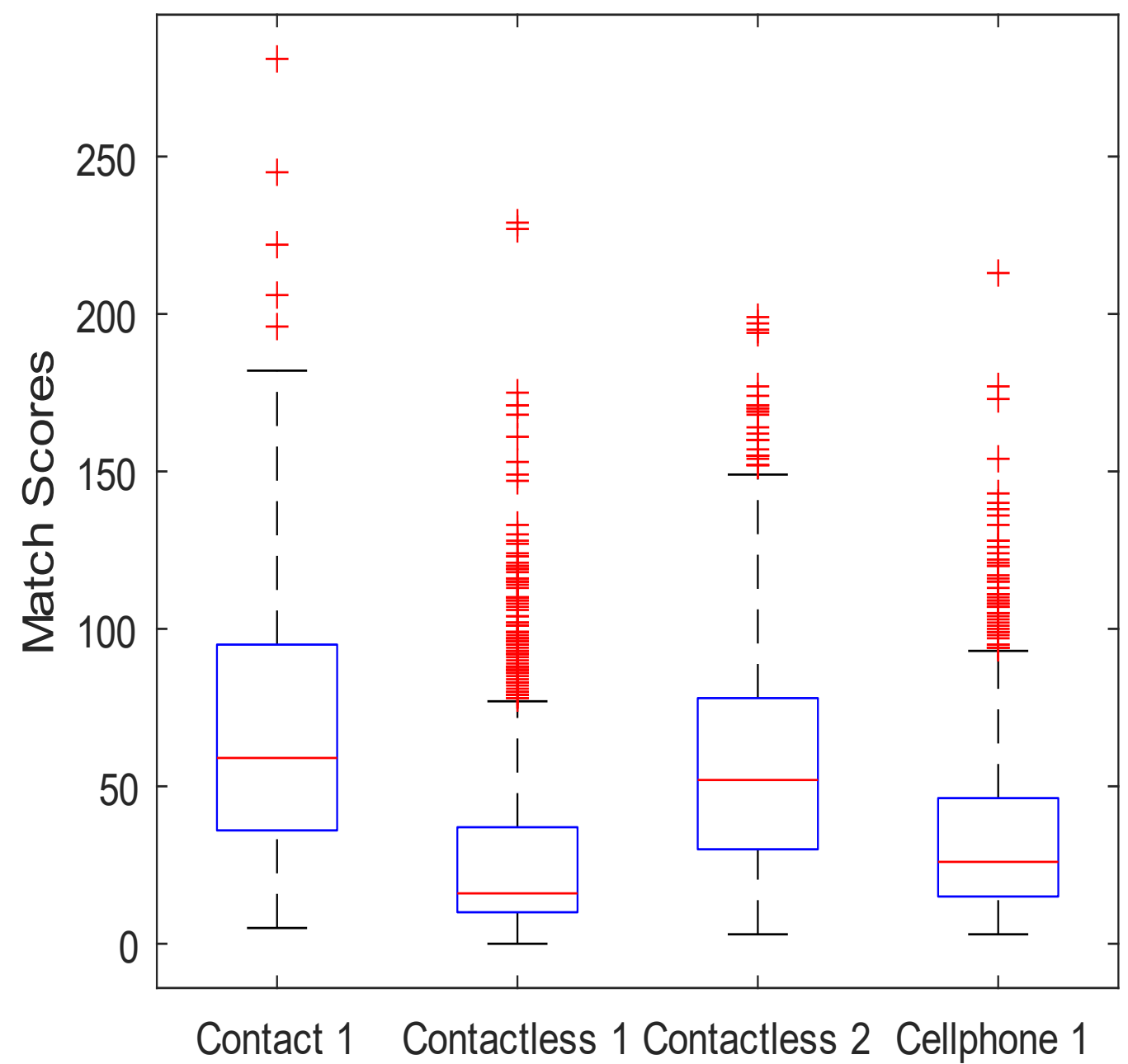

Figure 4.7: Comparison of the distribution of mated match scores for each device using Matcher-3.

The results shown in Figures 4.8-4.19 are distributions of mated match scores for each device on all three matchers. Each figure shows the distribution all matchers based on the recorded melanin values of the palm of the hand. For these distributions, the data is into three bins for each device. These bins separate the data based on the melanin measurement obtained from the skin reflectance data collected from the palm of the subjects. The threshold values used for these bins were calculated to split the groups into even ranges of melanin amounts.

For the melanin distributions, these plots show many outliers; however, the overall average area does not indicate a statistically relevant relationship between melanin content and match 
score. The plots for the melanin value lower than the first threshold do tend to have more outliers at the top end, however, it is apparent that the majority of the match scores fall within a similar range for all the data. As expected, the contact fingerprint matching data is clearly unaffected by the amount of melanin present.

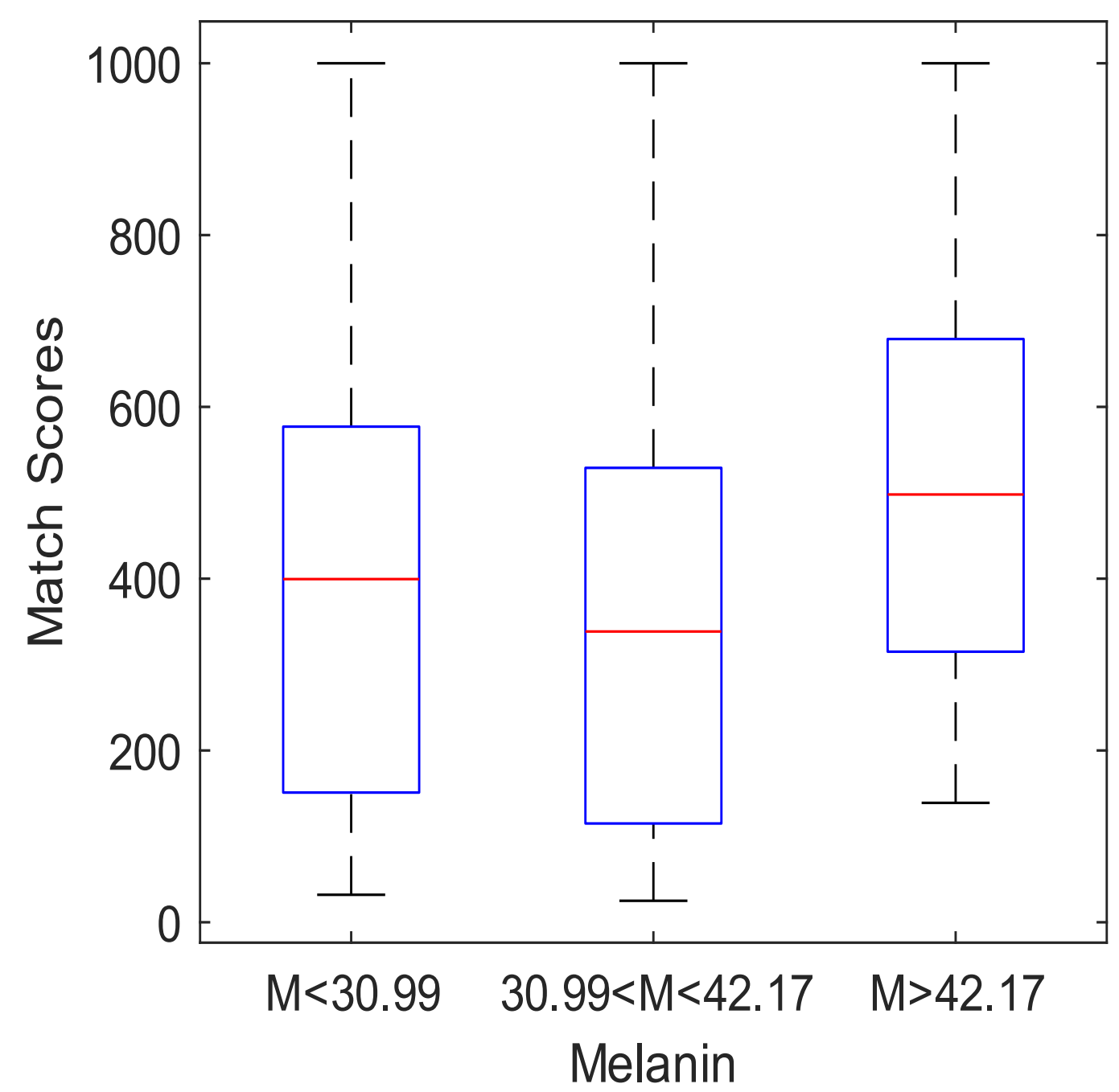

Figure 4.8: Comparison of the distribution of mated match scores based on melanin amount using probes from Contact-1 and Matcher-1. 


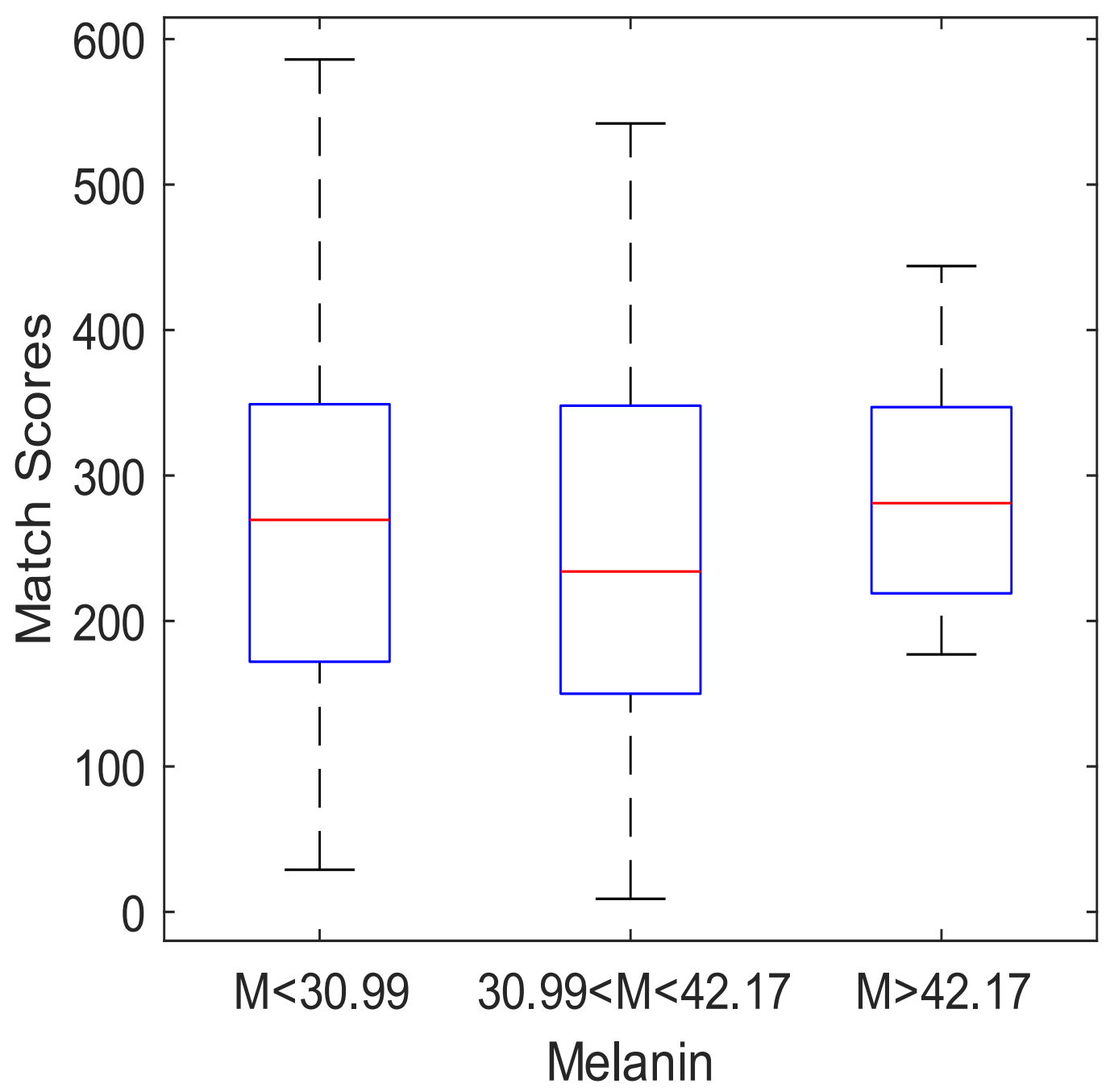

Figure 4.9: Comparison of the distribution of mated match scores based on melanin amount using probes from Contact-1 and Matcher-2. 


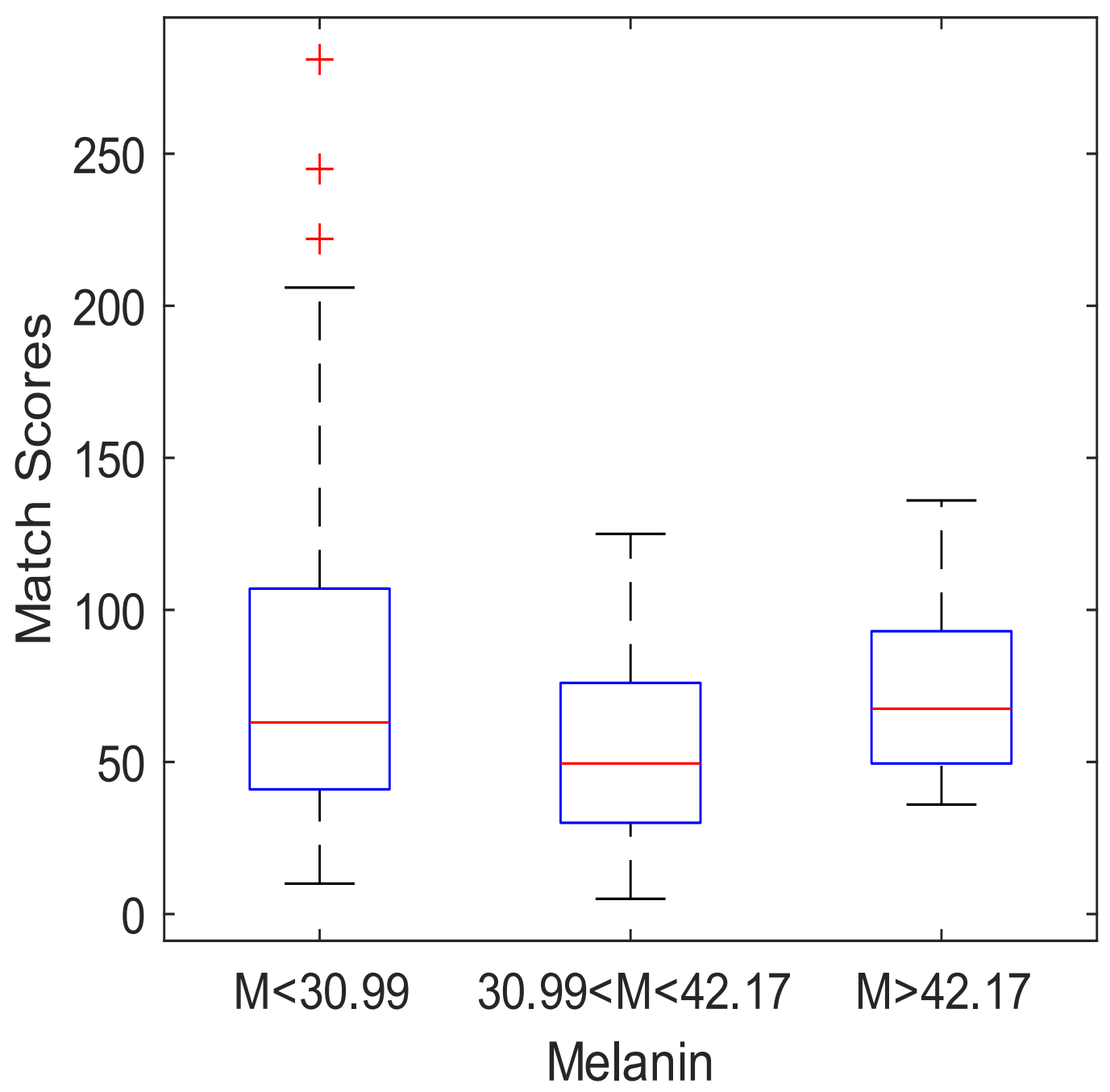

Figure 4.10: Comparison of the distribution of mated match scores based on melanin amount using probes from Contact-1 and Matcher-3. 


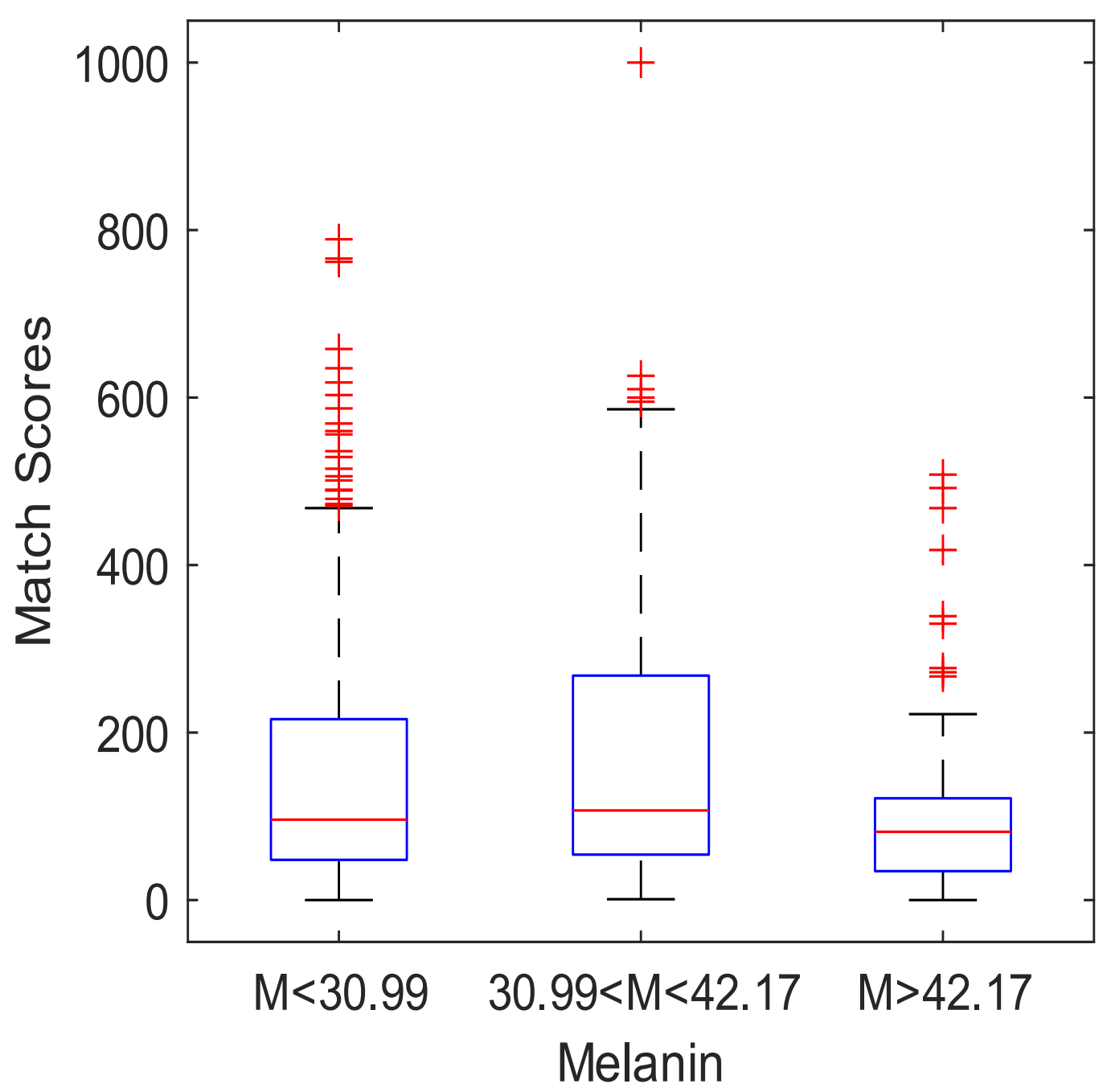

Figure 4.11: Comparison of the distribution of mated match scores based on melanin amount using probes from Contactless-1 and Matcher-1. 


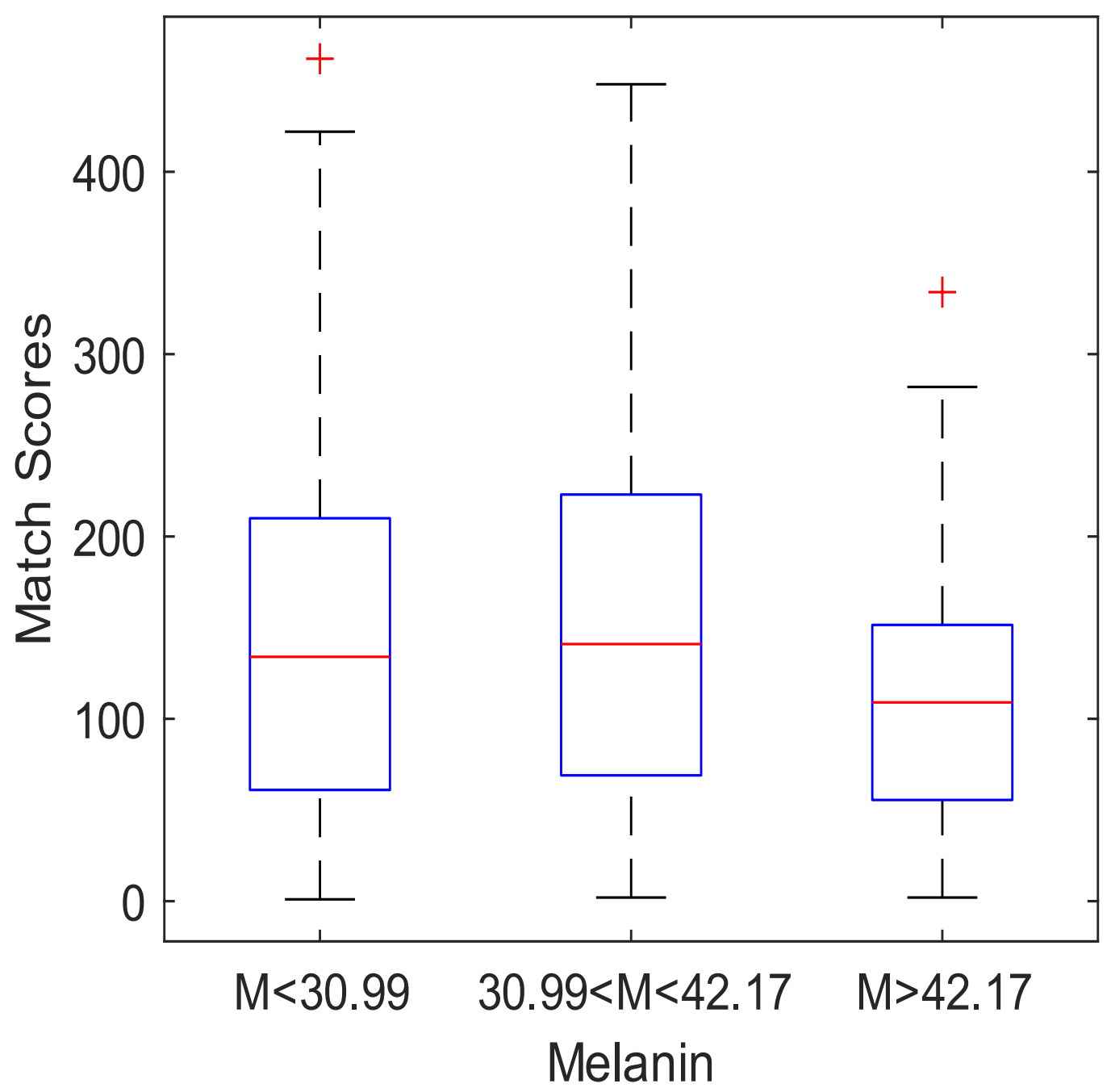

Figure 4.12: Comparison of the distribution of mated match scores based on melanin amount using probes from Contactless-1 and Matcher-2. 


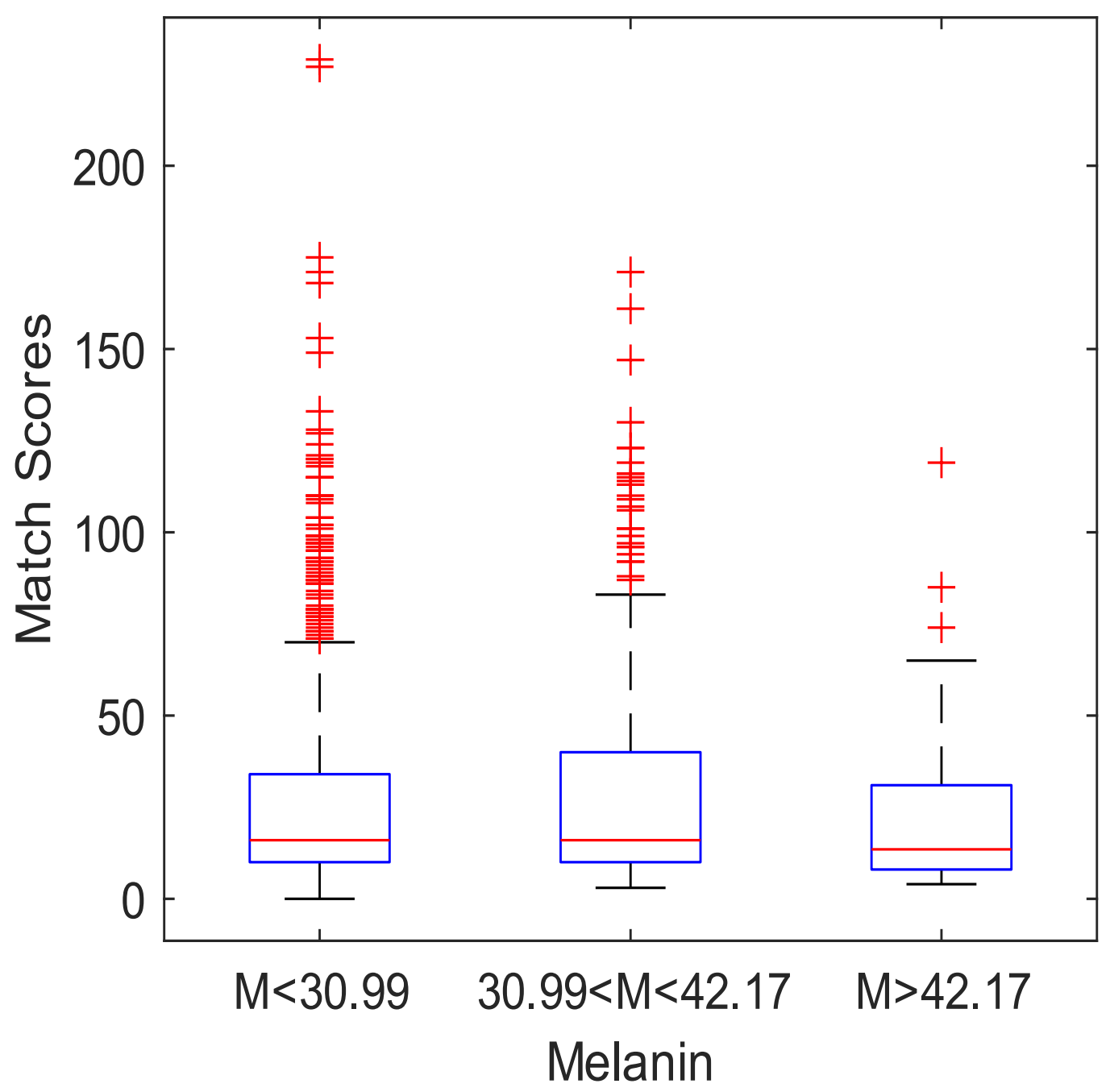

Figure 4.13: Comparison of the distribution of mated match scores based on melanin amount using probes from Contactless-1 and Matcher-3. 


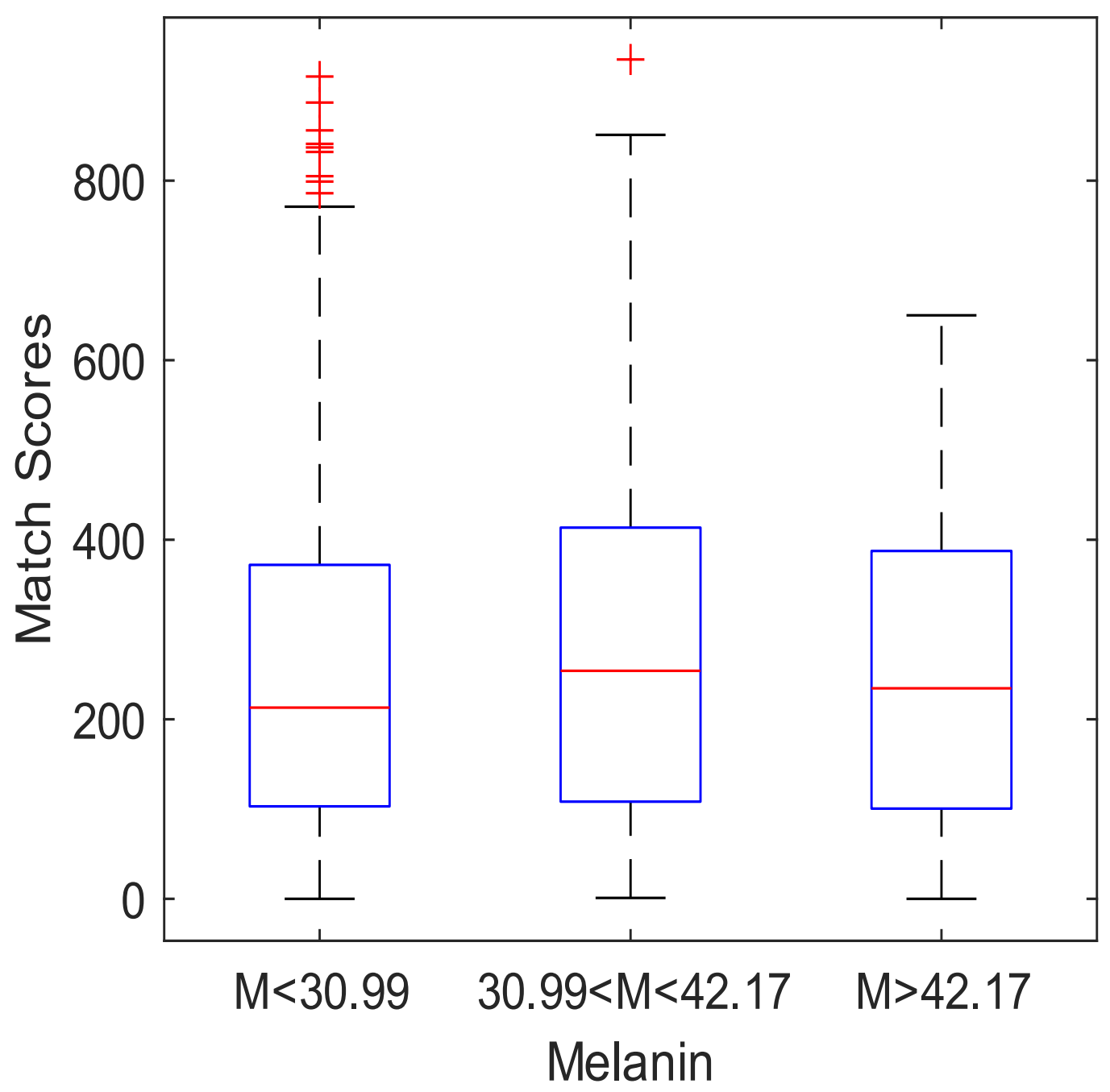

Figure 4.14: Comparison of the distribution of mated match scores based on melanin amount using probes from Contactless-2 and Matcher-1. 


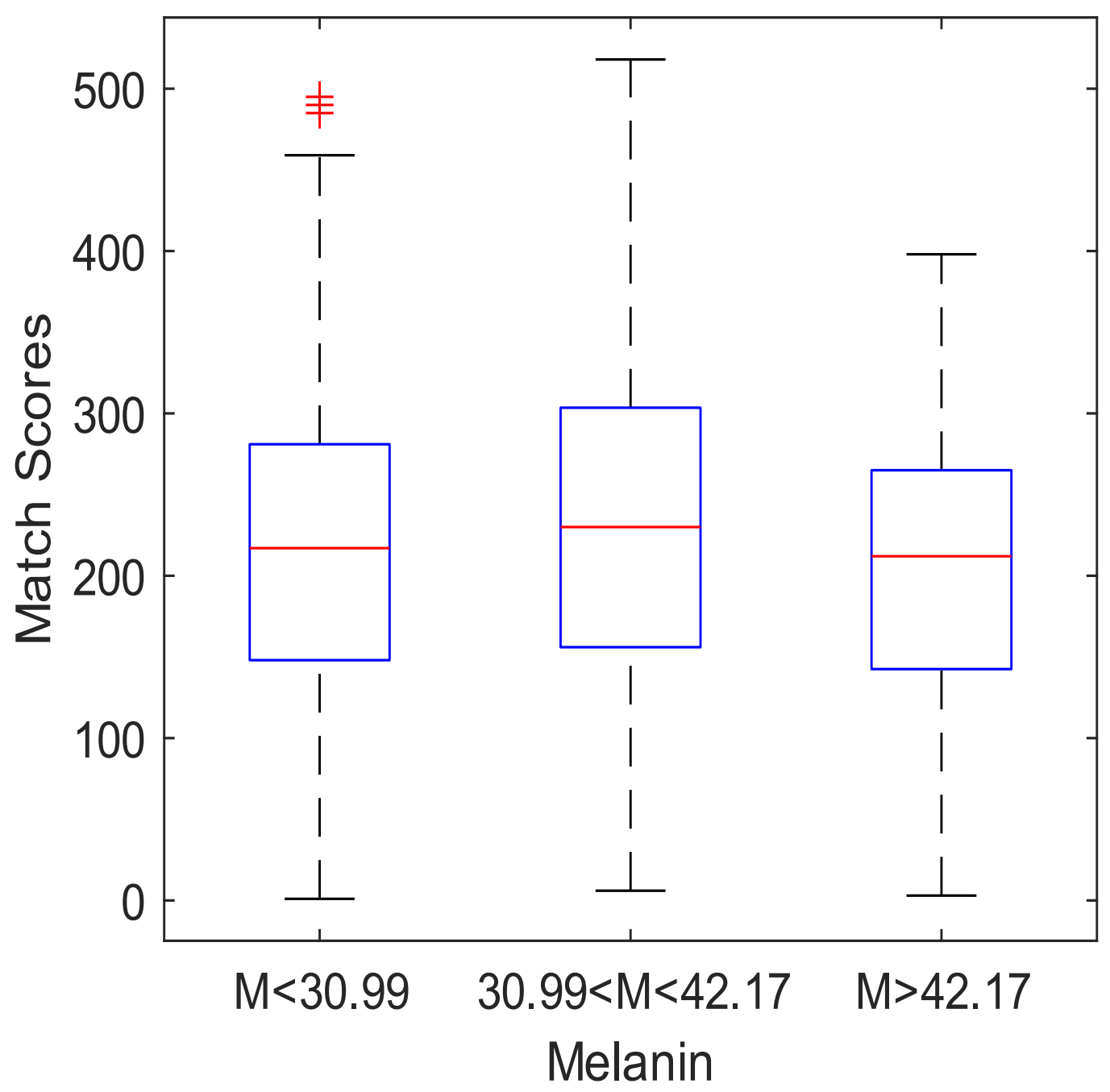

Figure 4.15: Comparison of the distribution of mated match scores based on melanin amount using probes from Contactless-2 and Matcher-2. 


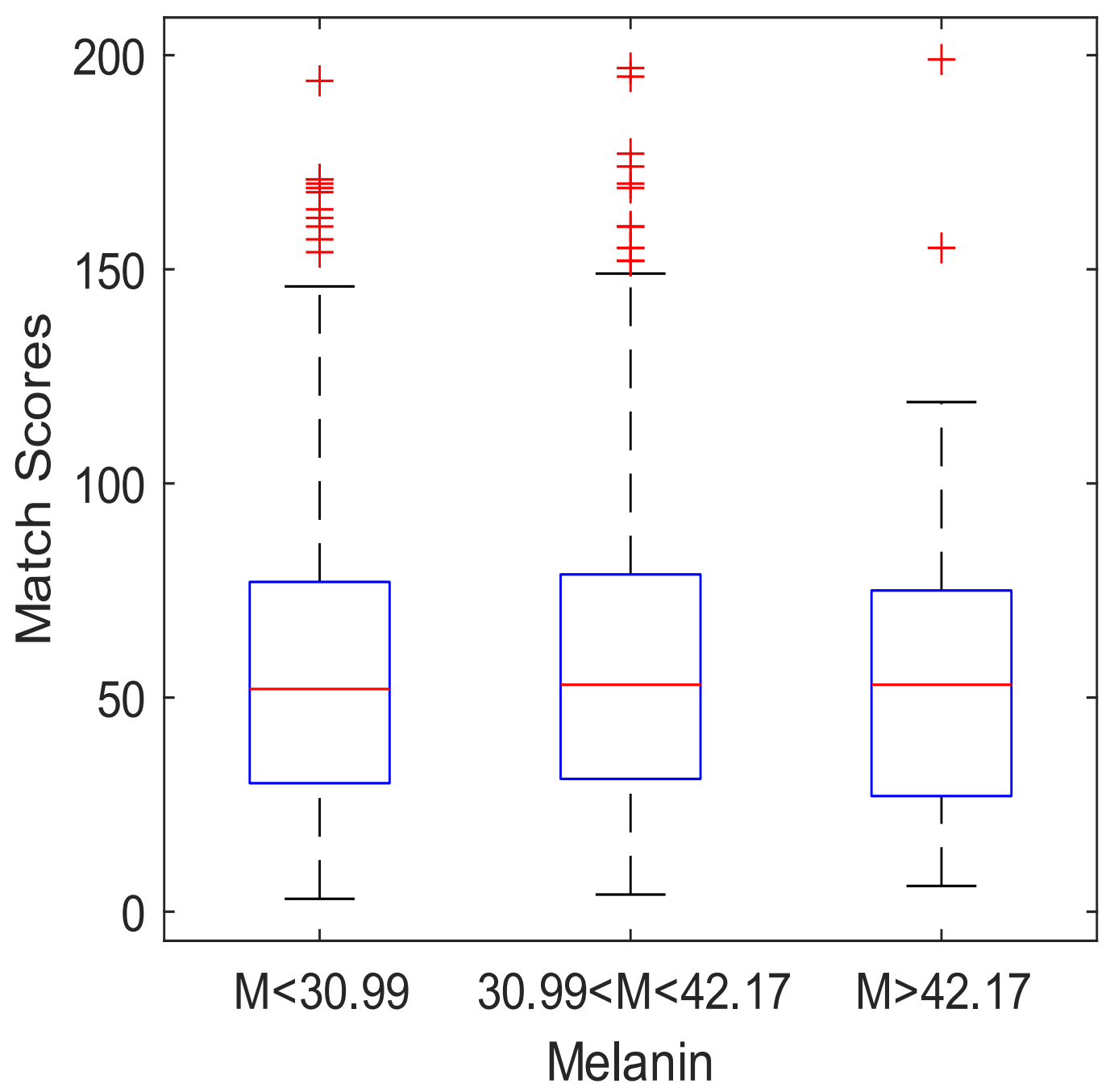

Figure 4.16: Comparison of the distribution of mated match scores based on melanin amount using probes from Contactless-2 and Matcher-3. 


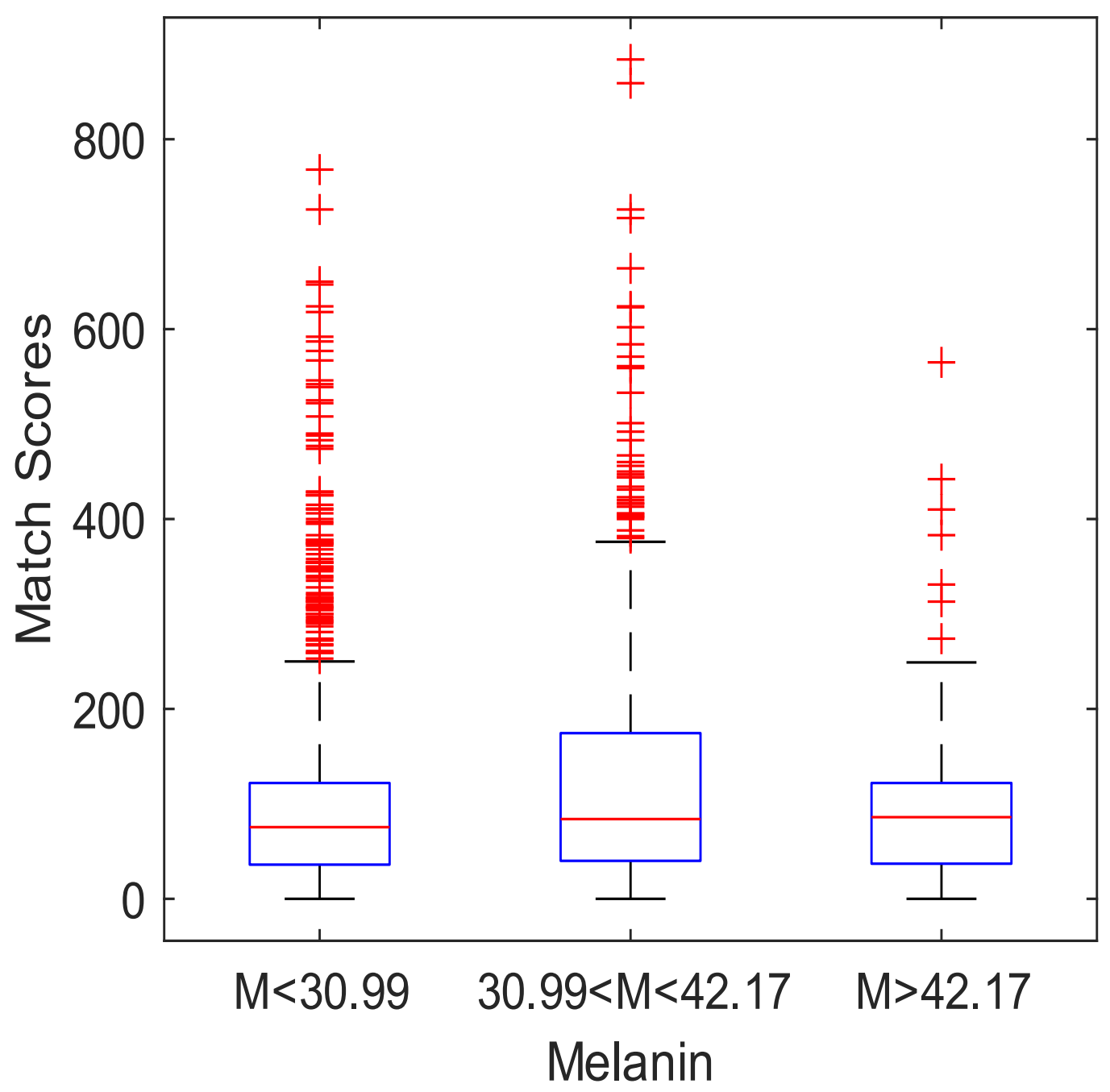

Figure 4.17: Comparison of the distribution of mated match scores based on melanin amount using probes from Cellphone-1 and Matcher-1. 


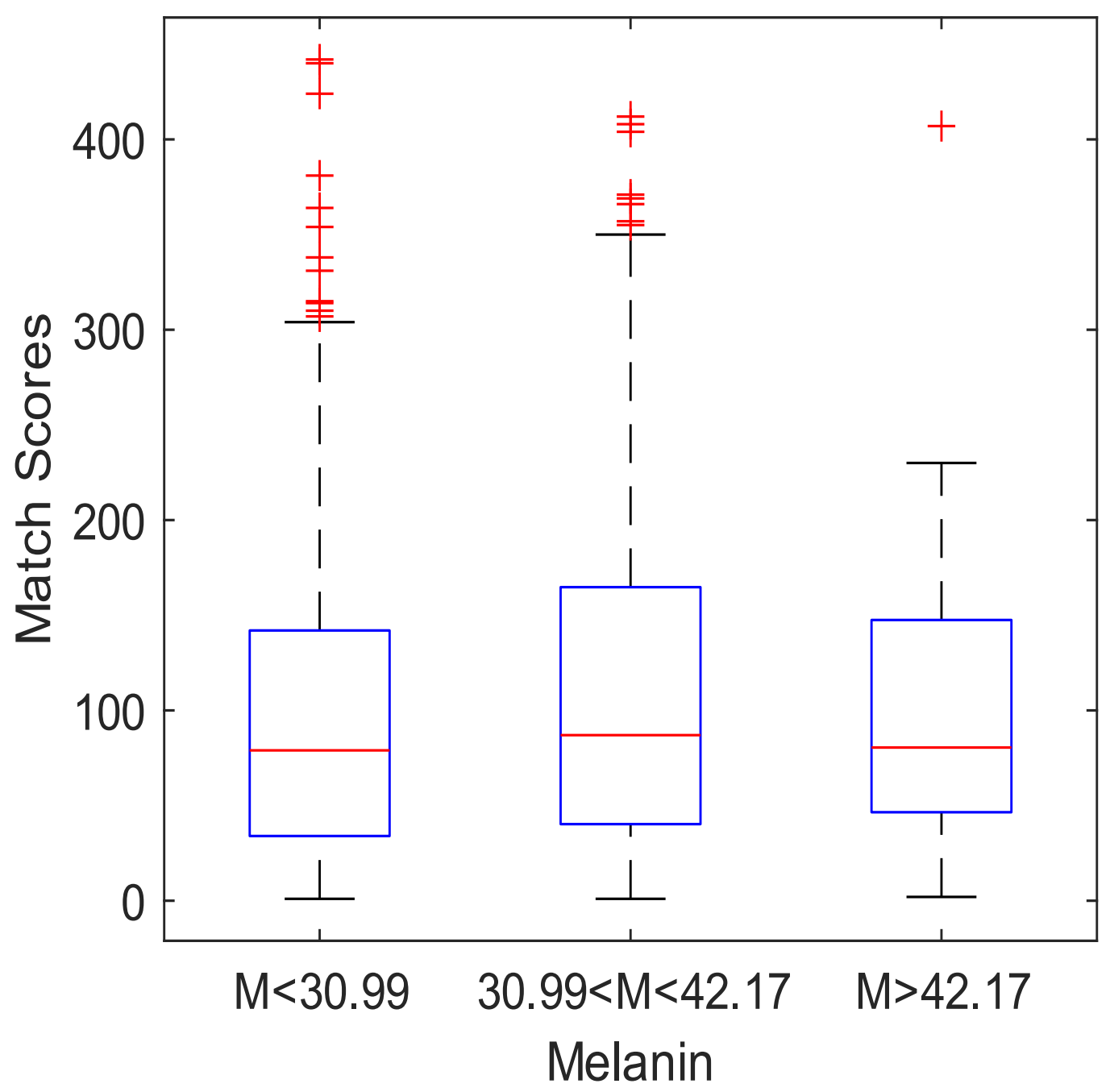

Figure 4.18: Comparison of the distribution of mated match scores based on melanin amount using probes from Cellphone-1 and Matcher-2. 


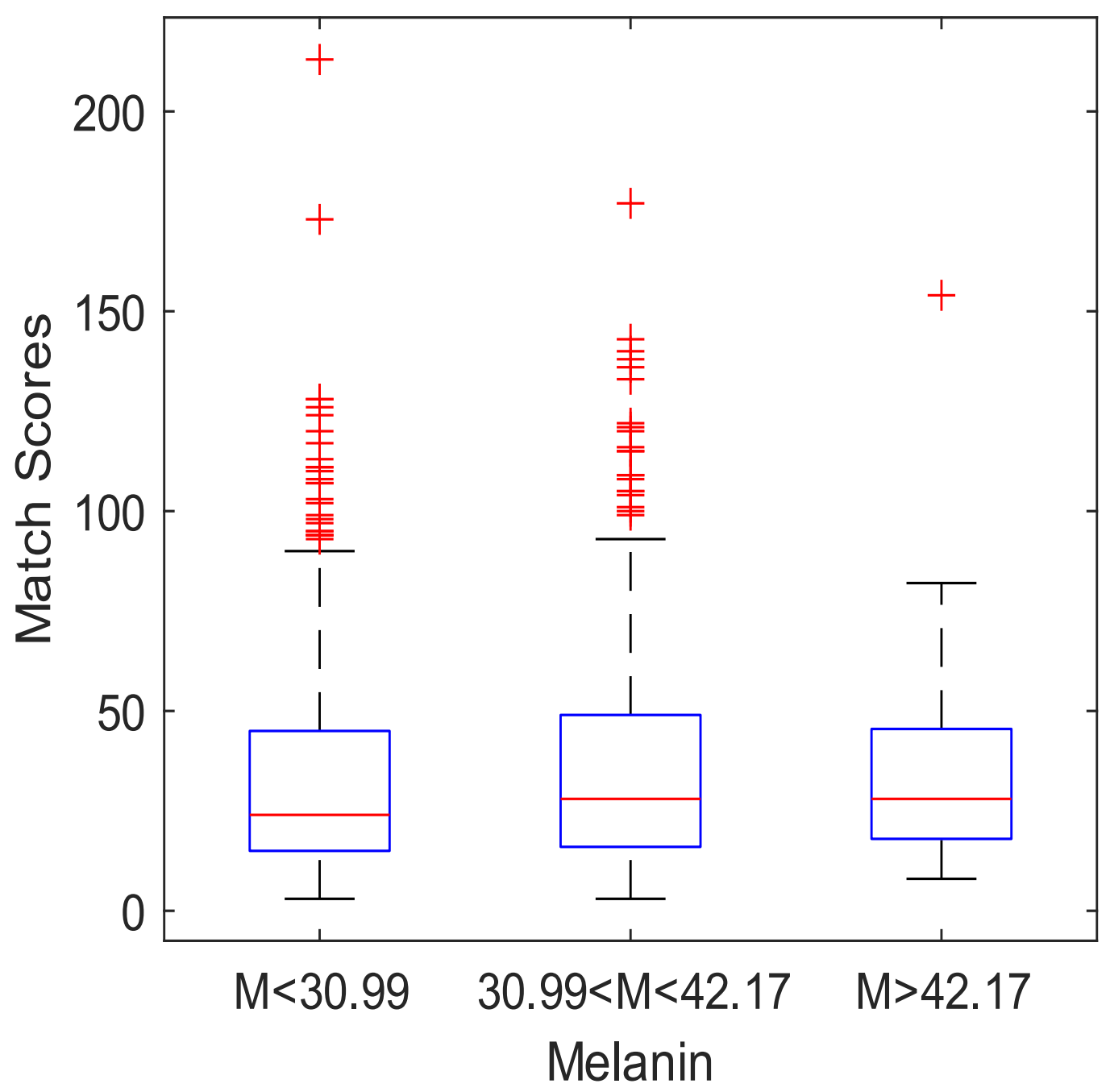

Figure 4.19: Comparison of the distribution of mated match scores based on melanin amount using probes from Cellphone-1 and Matcher-3.

The results shown in Figures 4.20-4.31 are distributions of mated match scores for each device on all three matchers. Each figure shows the distribution of scores based on the finger width values for one matcher. For these distributions, the data is sorted into three bins for each device. These bins separate the data based on the middle finger width calculated for each hand. The threshold values used for these bins were calculated to split the groups into even ranges of melanin amounts or finger width. 
Considering finger width distributions, the middle range of values from 30.99 to 42.17 has the highest-reaching whisker values. In terms of the overall results from this data, Matcher-1 was most affected by finger size for Contactless- 1 and Contactless-2, with larger sizes producing higher match scores. For images captured from the other devices, and all images on Matcher-2, there was no noticeable effect of finger width on match scores. For Matcher-3 there was no noticeable effect of the finger width on the matching performance. Along with the width analysis focused on the middle finger, an experiment was also performed using width data for the little finger of the right hand of all participants. The resulting match score distributions showed similar results to the middle finger values, and thus, were not included here.

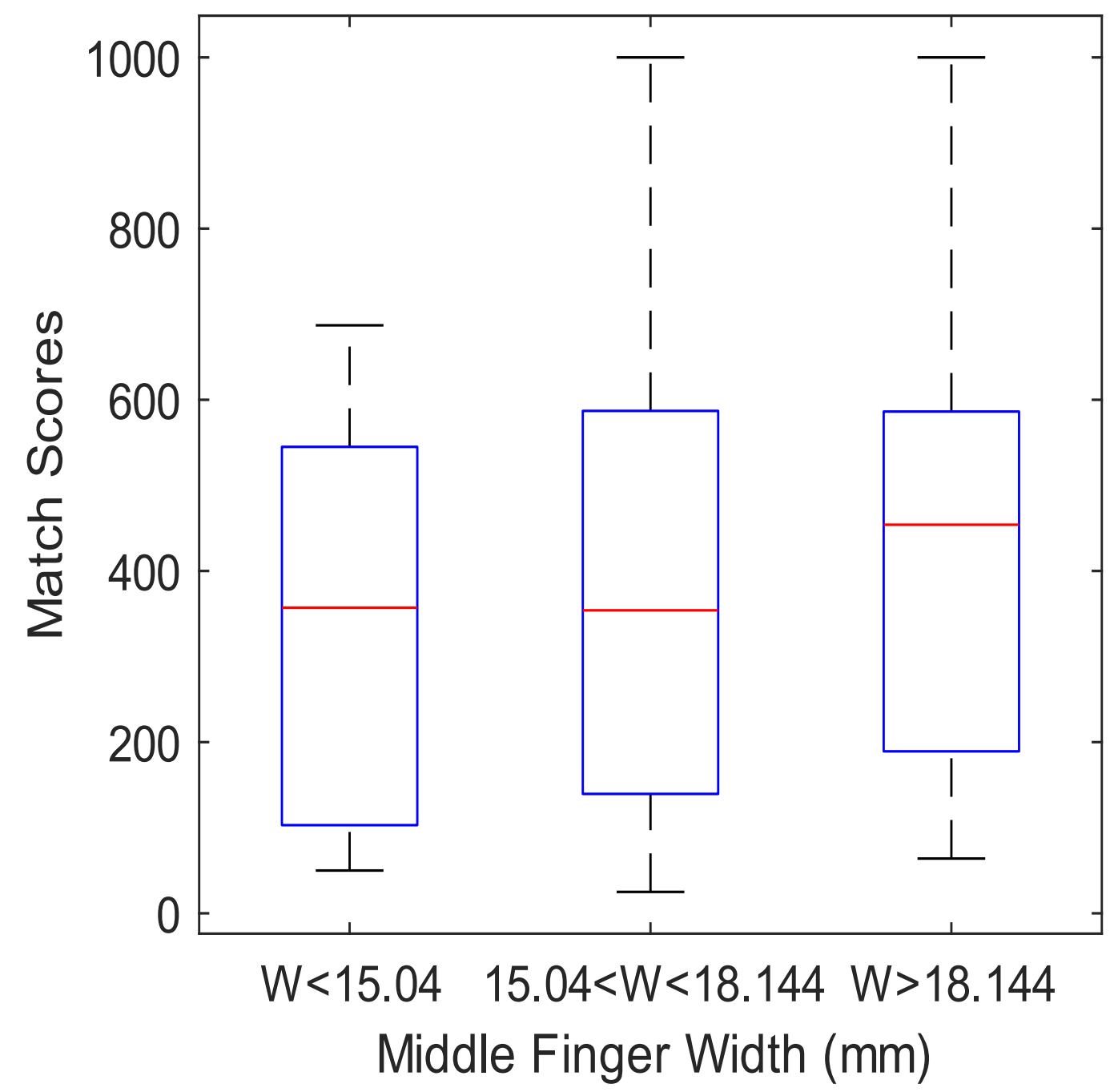

Figure 4.20: Comparison of the distribution of mated match scores based on middle finger width using probes from Contact-1 and Matcher-1. 


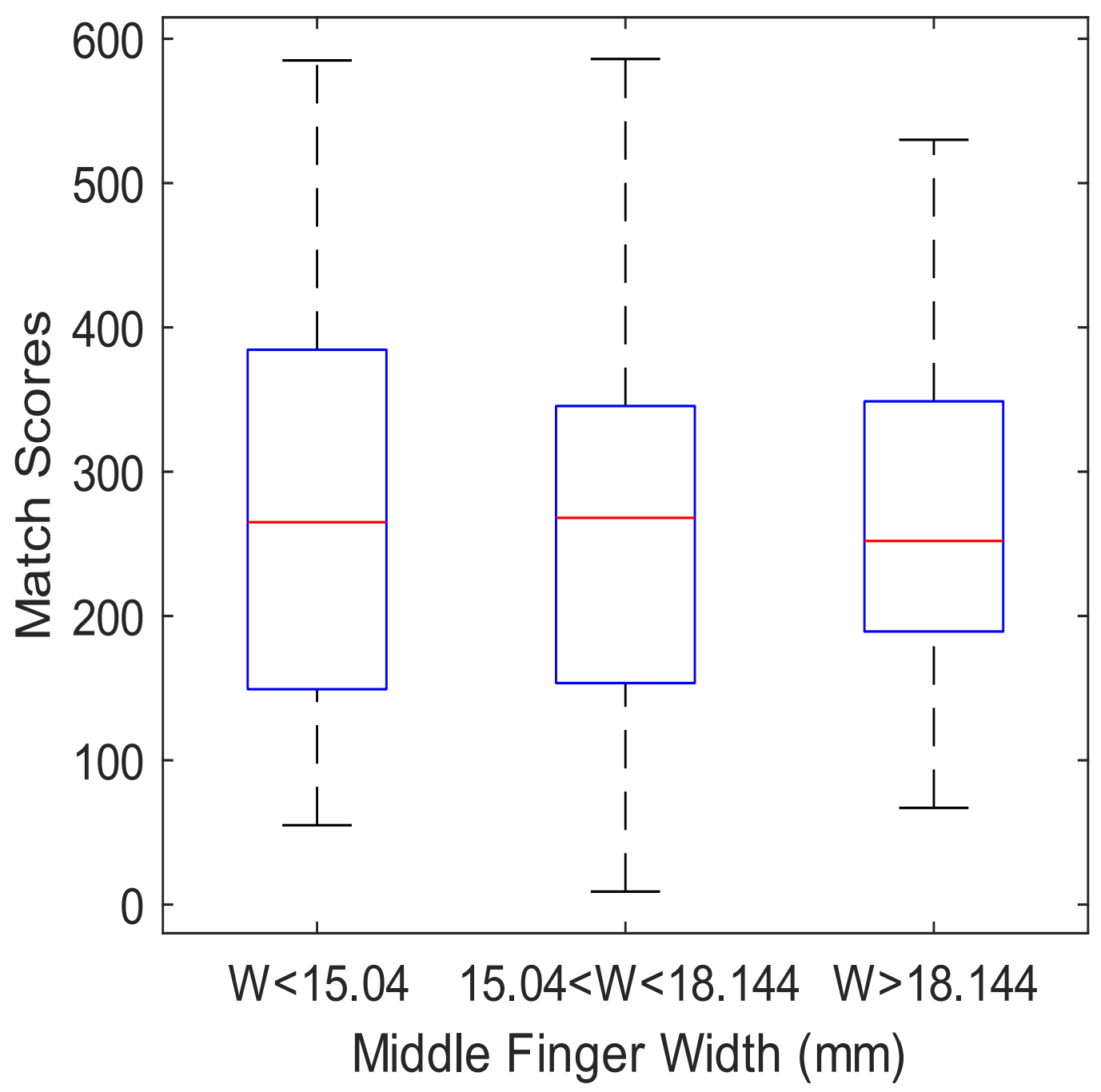

Figure 4.21: Comparison of the distribution of mated match scores based on middle finger width using probes from Contact-1 and Matcher-2. 


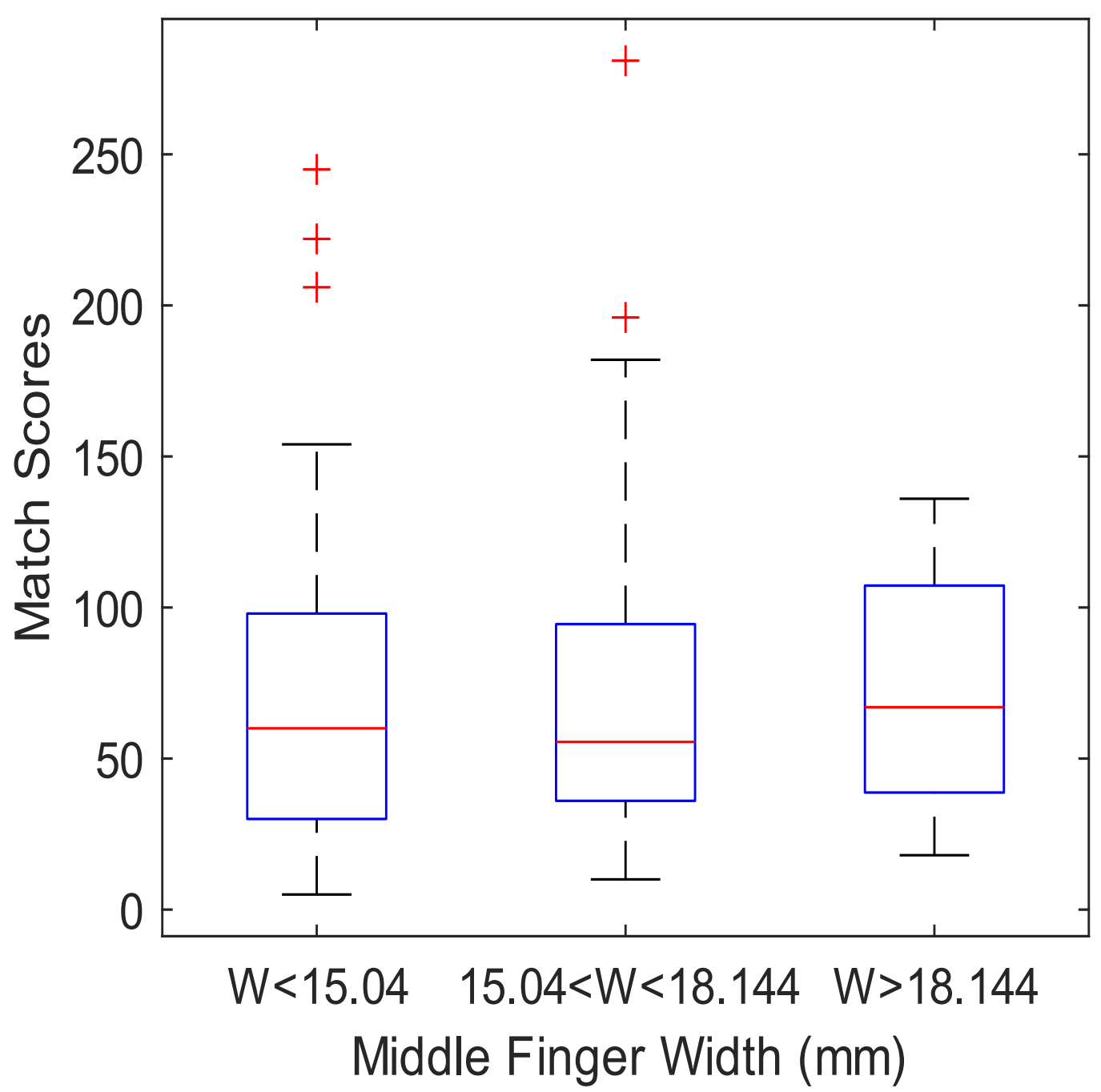

Figure 4.22: Comparison of the distribution of mated match scores based on middle finger width using probes from Contact-1 and Matcher-3. 


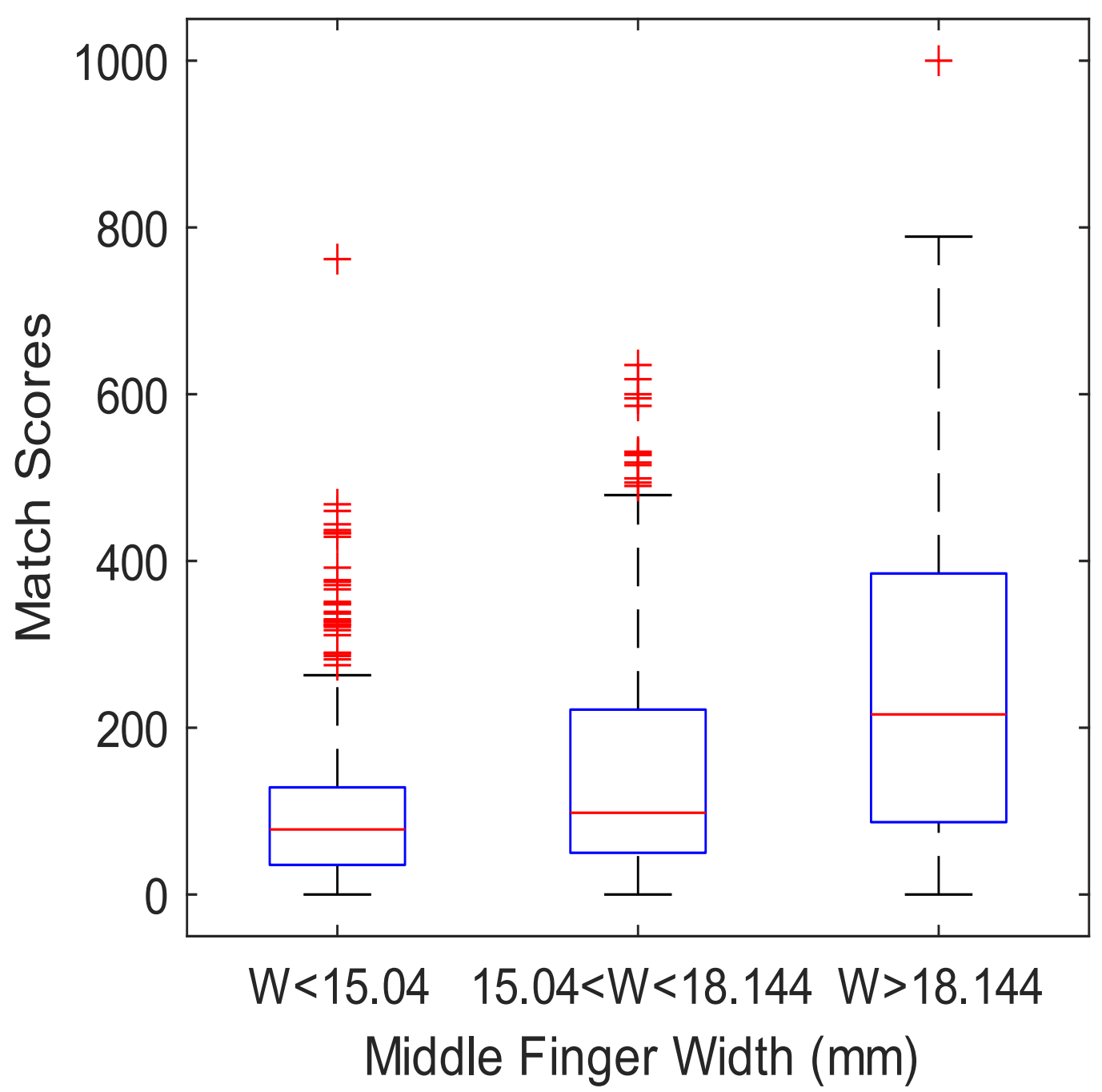

Figure 4.23: Comparison of the distribution of mated match scores based on middle finger width using probes from Contactless-1 and Matcher-1. 


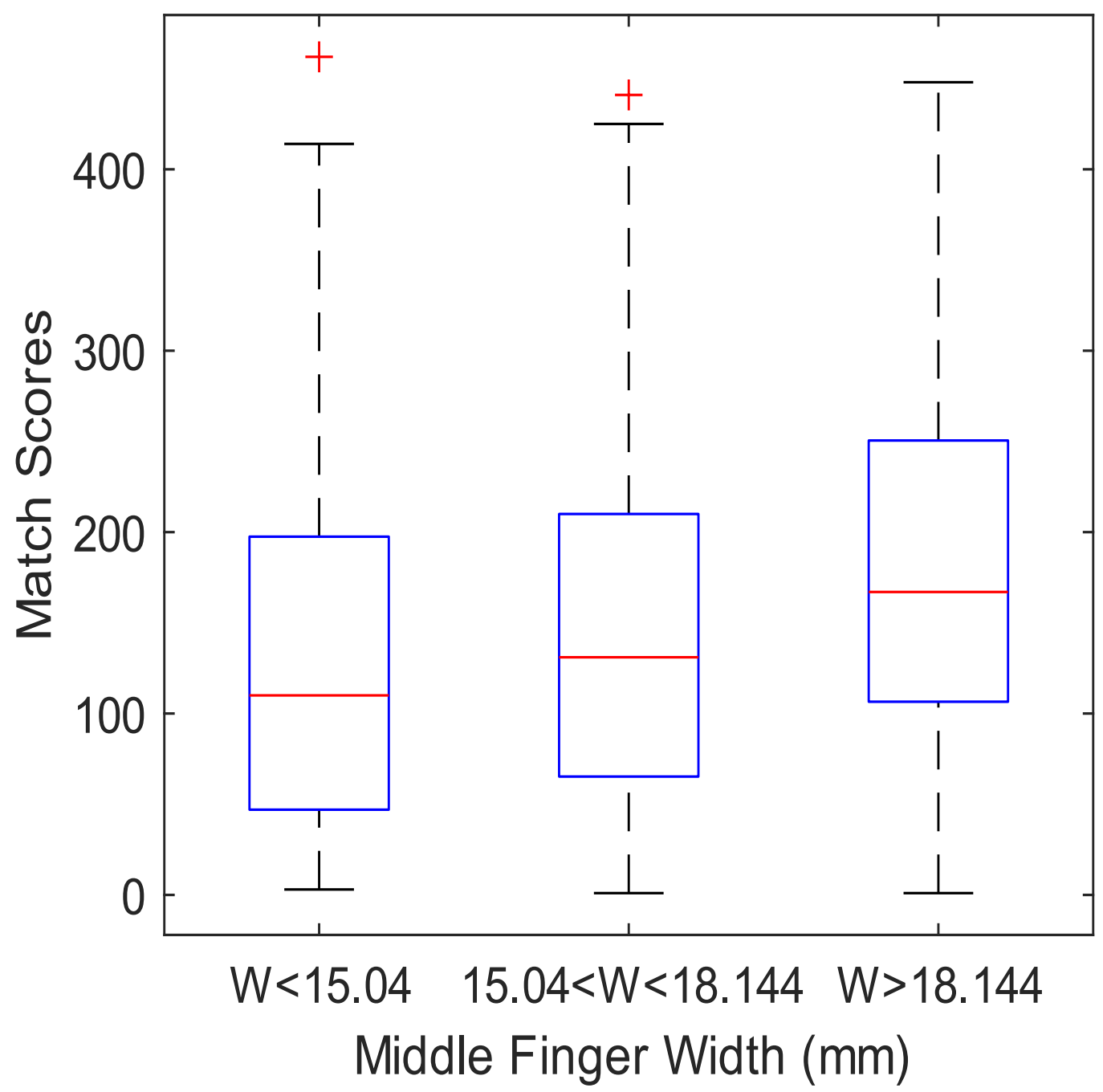

Figure 4.24: Comparison of the distribution of mated match scores based on middle finger width using probes from Contactless-1 and Matcher-2. 


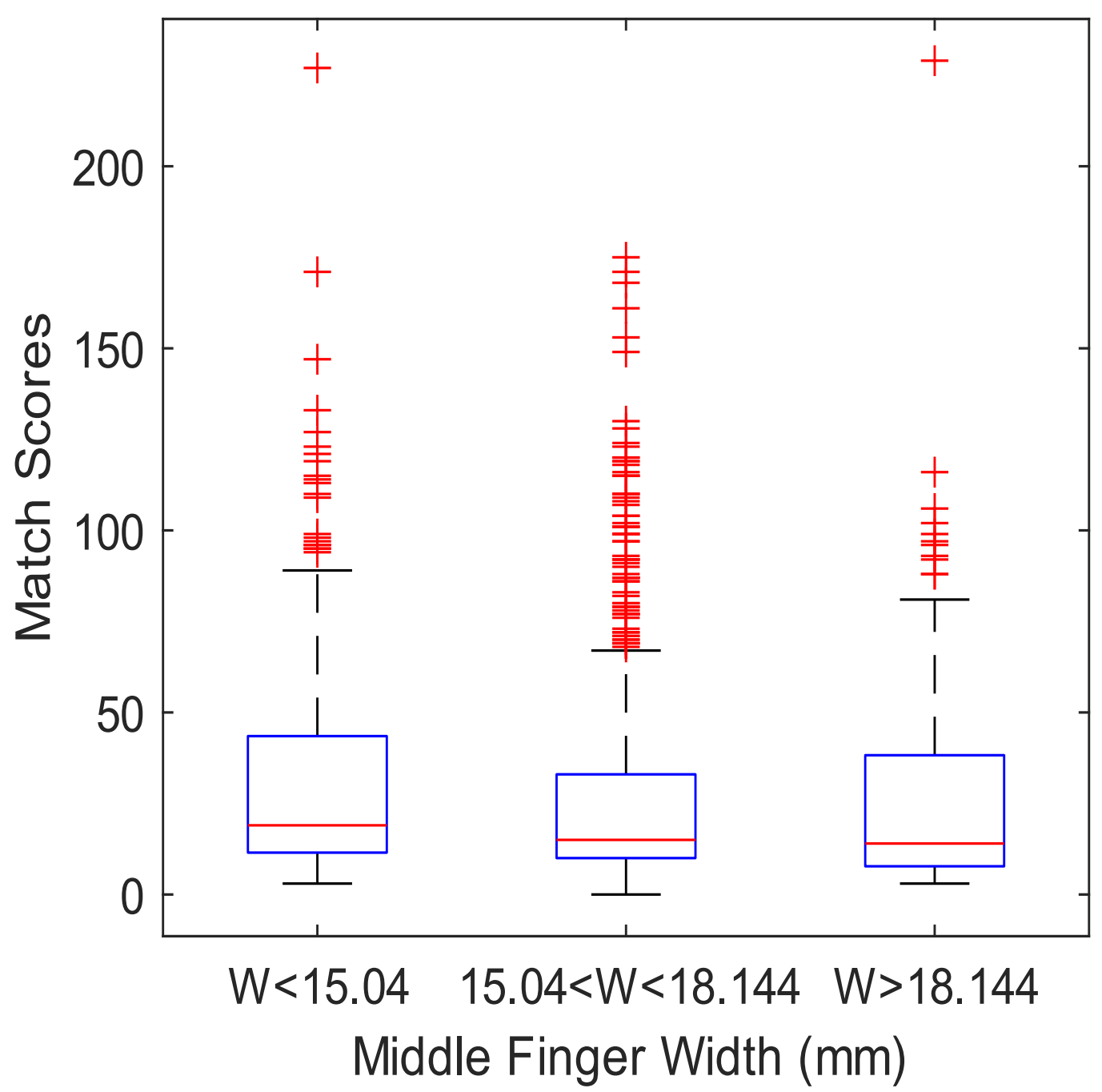

Figure 4.25: Comparison of the distribution of mated match scores based on middle finger width using probes from Contactless-1 and Matcher-3. 


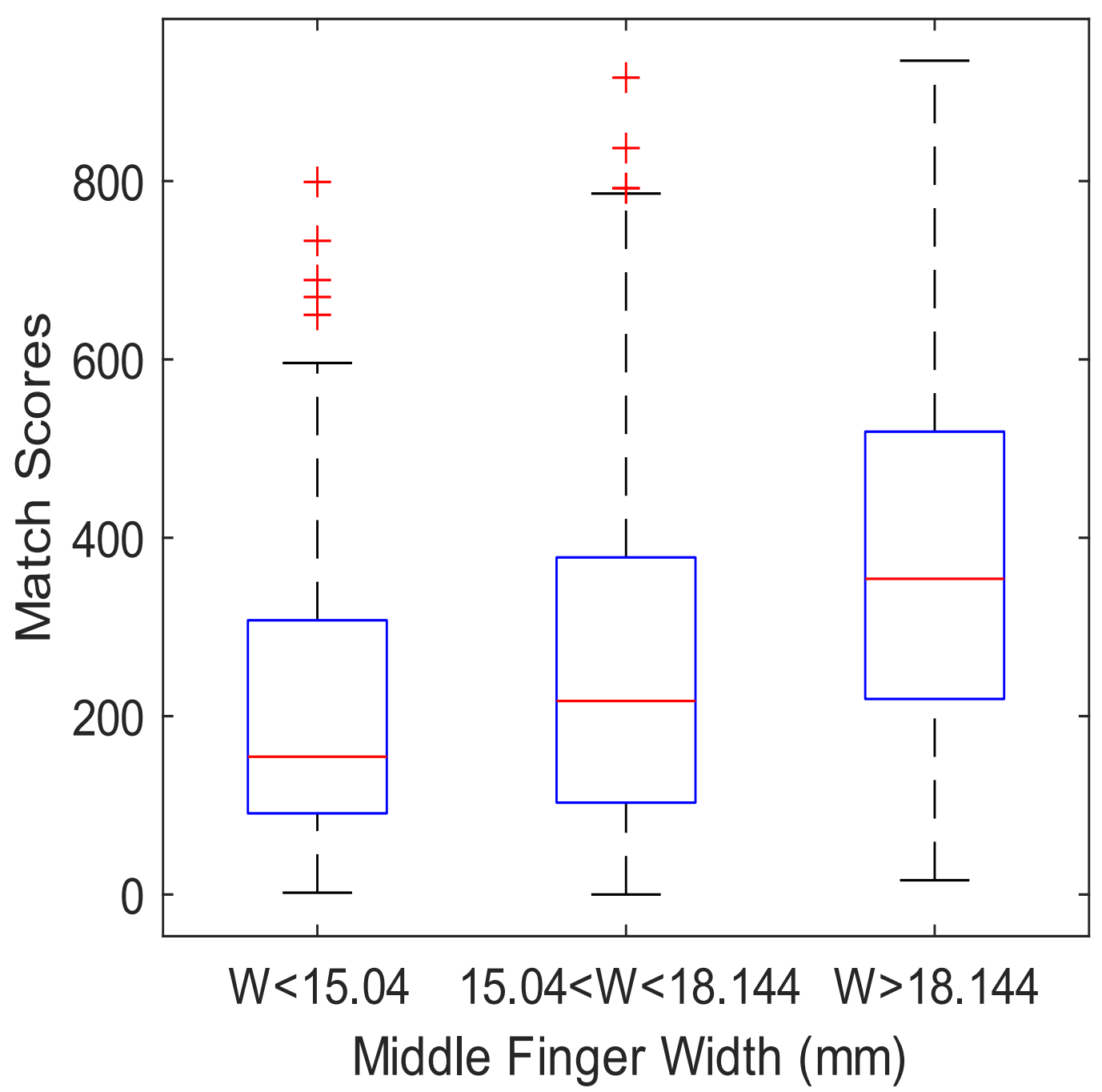

Figure 4.26: Comparison of the distribution of mated match scores based on middle finger width using probes from Contactless-2 and Matcher-1. 


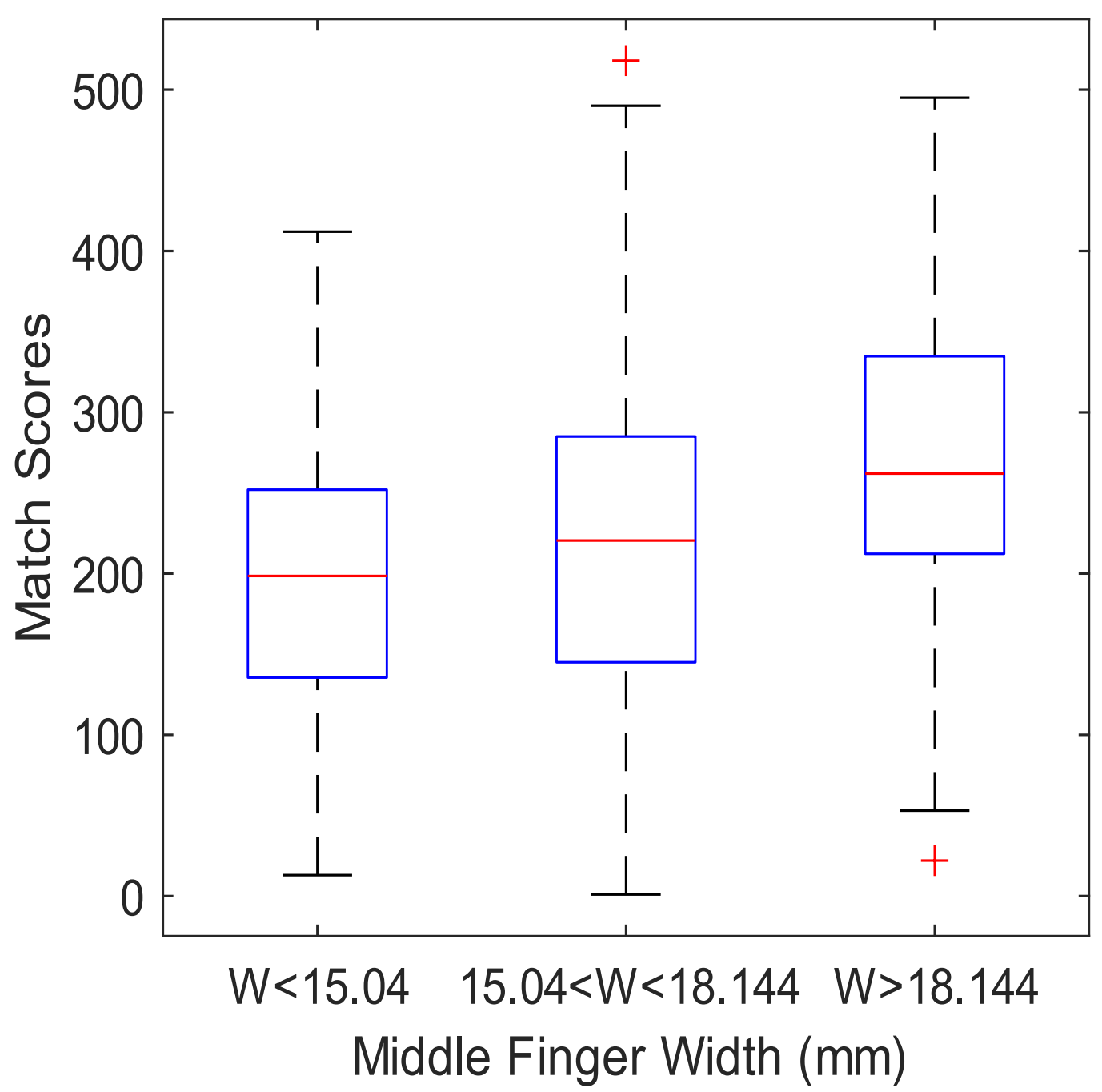

Figure 4.27: Comparison of the distribution of mated match scores based on middle finger width using probes from Contactless-2 and Matcher-2. 


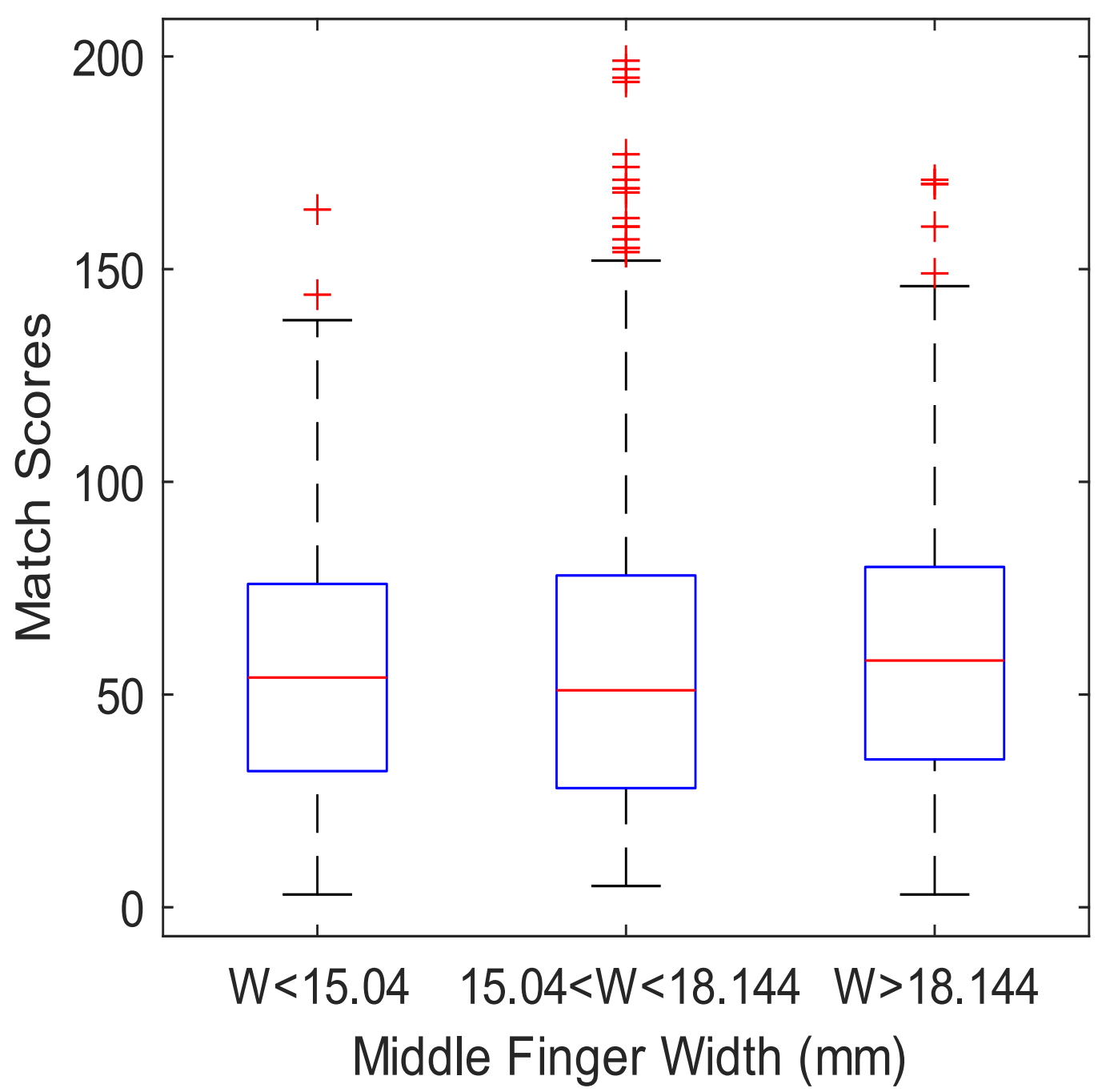

Figure 4.28: Comparison of the distribution of mated match scores based on middle finger width using probes from Contactless-2 and Matcher-3. 


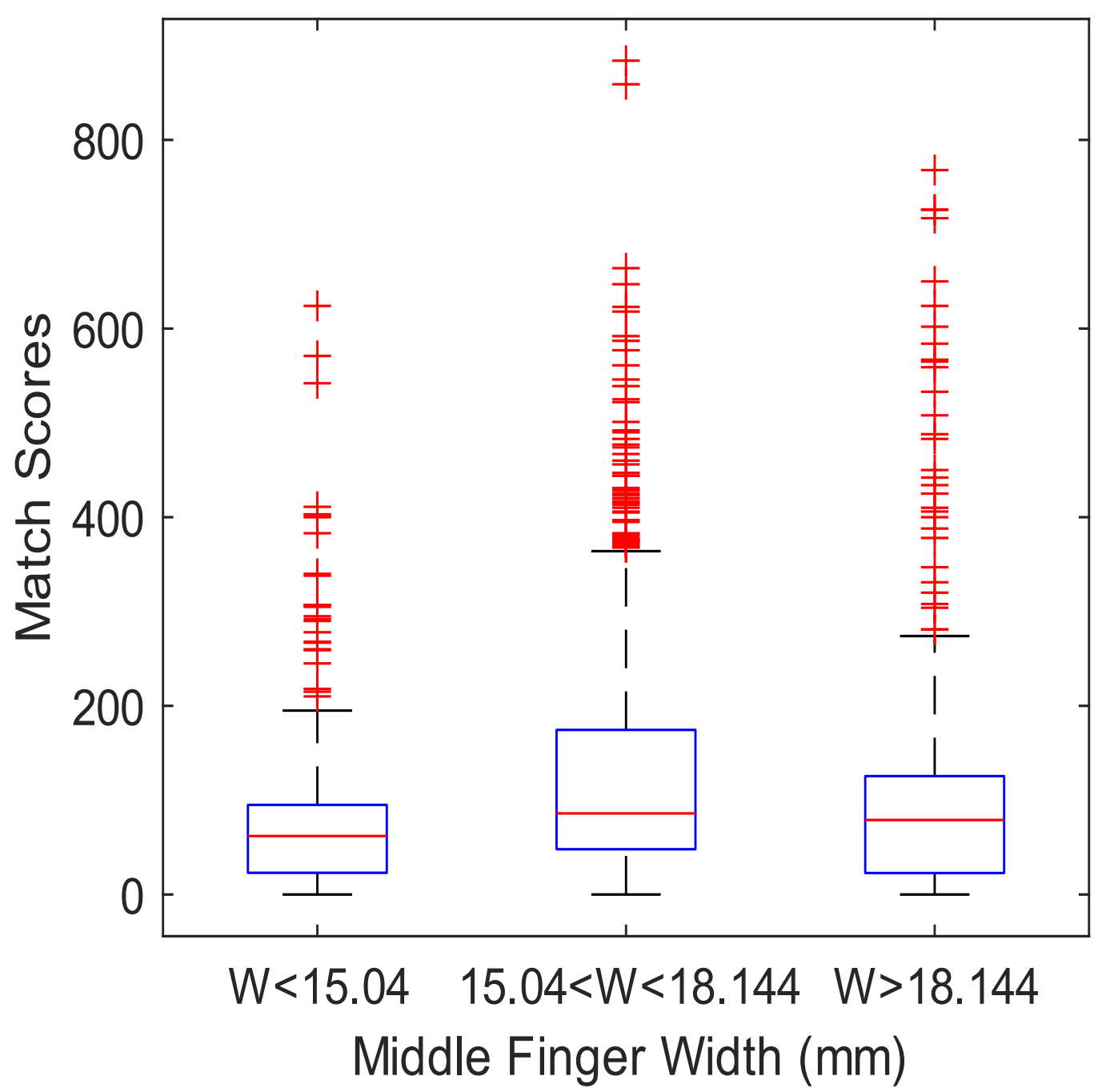

Figure 4.29: Comparison of the distribution of mated match scores based on middle finger width using probes from Cellphone-1 and Matcher-1. 


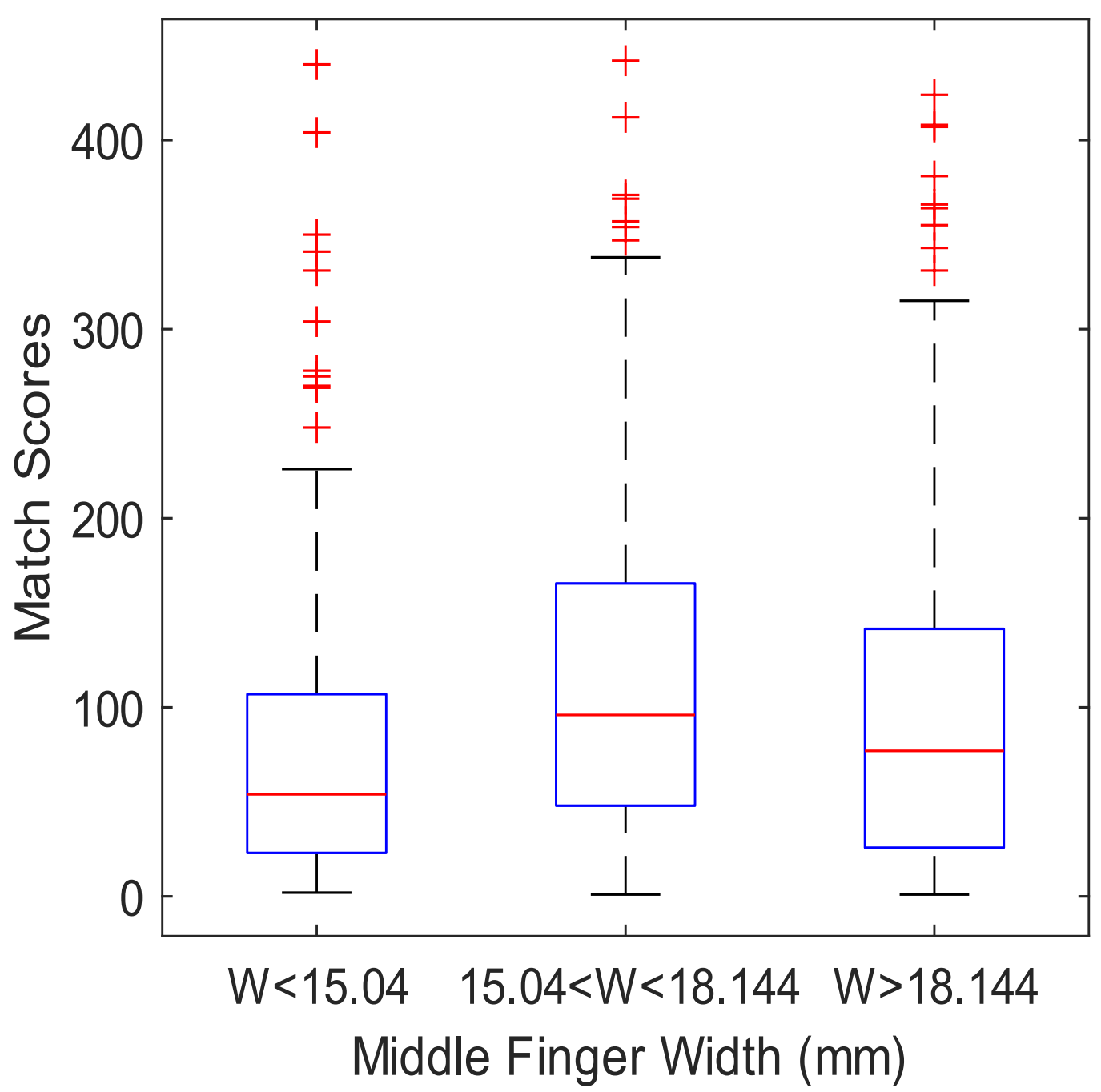

Figure 4.30: Comparison of the distribution of mated match scores based on middle finger width using probes from Cellphone-1 and Matcher-2. 


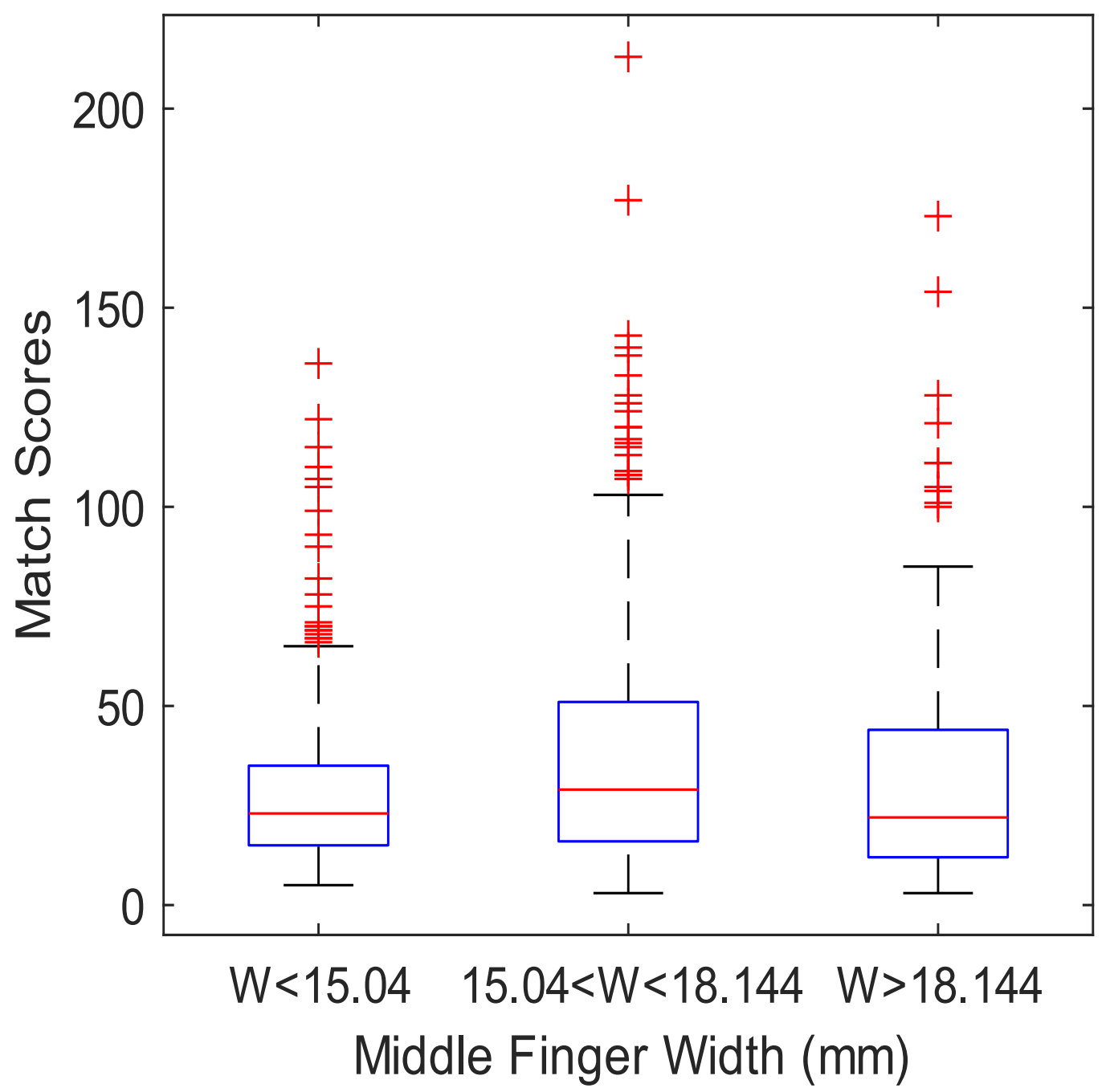

Figure 4.31: Comparison of the distribution of mated match scores based on middle finger width using probes from Cellphone-1 and Matcher-3. 


\section{Chapter 5: \\ Conclusion and Future Work}




\subsection{Conclusion}

This work explored the interoperability of fingerprints captured from multiple contactless fingerprint devices matched against a gallery of fingerprints captured using a contact-based fingerprint device. Based on the results shown, the Contactless-2 device outperformed both Contactless-1 and Cellphone-1, with an AUC of 0.9818, 0.9955, and 0.9551 for Matcher-1, Matcher-2, and Matcher-3, respectively, with the latter two performing within 0.0176 and 0.0185 of each other for Matcher-1 and Matcher-2, respectively. Using Matcher-3, Contactless-1 fell below Cellphone-1 by a margin of 0.1709 . Again, this performance is likely due to the lack of optimization done for Matcher-3. The Cellphone-1 images outperformed the baseline Cellphone1 -raw images by a margin of $0.1512,0.2213,0.2642$ based on the AUC, as expected.

After the matching analysis was completed, an evaluation of the impact of skin color, collected via skin reflectometer, on match performance was conducted. From this skin reflectance data, a measure of the melanin present in the palm of the subjects was used to split the match scores into groups. This was used to generate new distributions to show the performance for each group. Based on these distributions of the results, there was no perceivable impact across all the experiments based on statistical significance. This also shows that the contact-based fingerprints were unaffected by melanin content, as was the expected outcome.

A similar analysis was performed for finger size using the width of the middle finger from the right hand of each participant. Again, the data was split into groups based on finger width data, and the match results were used to generate a distribution to convey the performance of the matching based on the various widths. In this case, there was a noticeable effect on the match scores of Contactless- 1 and Contactless- 2 when using Matcher-1. This effect was not present in either Contact-1 or Cellphone-1 images used as probes to the same matcher, nor was it observed with probes images from any of the devices matched by Matcher-2 or Matcher-3.

Based on the results of this work, it has been shown that contactless fingerprint devices, such as Contactless-2, can achieve a match performance approaching that of contact fingerprints. In comparison to previous work from [3], Cellphone-1 with an AUC of 0.9764, 0.9635, and 0.8606 from Matcher-1, Matcher-2, and Matcher-3 respectively outperforms similar cellphone-based device performance. As well, Contactless-1, while not matching the results of Contactless-2, exceeds the match performance results of many of the devices from [3] as well. 
Based on the results of this work, it appears that contactless fingerprint solutions are improving in the area of interoperability.

\subsection{Future Work}

Given that the want and need for convenient and hygienic methods of fingerprint collection are ever-present and increasing due to outside influences such as the COVID-19 pandemic, contactless fingerprinting will continue to grow as an area for collecting fingerprints. This continually increasing need will require continued study into the usability of fingerprints gathered in a contactless manner given that the legacy data was collected from contact-based methods.

Based on the work presented further studies on the interoperability of contactless and contact fingerprinting can build upon the results presented, as these results built upon previous studies. Along with further interoperability studies, continued studies into the effect of skin reflectance values and finger width can be performed with more participants and other fingerprinting devices to expand upon the results shown here. The continued study of the effect of skin reflectance on match performance can include studying other techniques for measuring skin reflectance and other color spaces that may provide further results and insight. Continuing the focus on finger size can gather more data and tie into the methods used in hand geometry studies to further provide results and conclusions on the effect of finger size for matching both in contact and contactless devices. 


\section{References}

[1] D. Maltoni, D. Maio, A. K. Jain and S. Prabhakar, Handbook of Fingerprint Recognition, Springer, 2014.

[2] J. Libert, J. Grantham, B. Bandini, S. Wood, M. Garris, K. Ko, F. Byers and C. Watson, "Guidance for Evaluating Contactless Fingerprint Acquisition Devices," National Institute of Standards and Technology, 2018.

[3] J. Libert, J. Grantham, B. Bandini, K. Ko, S. Orandi and C. Watson, "Interoperability Assessment 2019: Contactless-to-Contact Fingerprint Capture," National Institute of Standards and Technology, 2020.

[4] J. Priesnitz, C. Rathgeb, N. Buchmann and et al., "An Overview of Touchless 2D Fingerprint Recognition," EURASIP Journal on Imageand Video Processing, no. 8 (2021), 2021.

[5] "Identification and Authentication within Reach," Thales Group. [Online]. [Accessed March 2021].

[6] "MorphoWave Compact," Idemia. [Online]. [Accessed March 2021].

[7] "TBS 3D Terminal," TBS. [Online]. [Accessed March 2021].

[8] A. K. Jain, A. Ross and S. Prabhakar, "An Introduction to Biometric Recognition," IEEE Transactions on Circuits and System for Video Technology, vol. 14, no. 1, pp. 4-20, 2004.

[9] K. Mangold, "Data Format for the Interchange of Fingerprint, Facial \& Other Biometric Information ANSI/NIST-ITL 1-2011 NIST Special Publication 500-290 Edition 3," National Institute of Standards and Technology, 2016.

[10] J. Priesnitz, C. Rathgeb, N. Buchmann and C. Busch, "Touchless Fingerprint Sample Quality: Prerequisites for the Applicability of NFIQ2.0," in International Conference of the Biometrics Special Interest Group (BIOSIG), Darmstadt, Germany, 2020. 
[11] E. Biller, "Interoperability Analysis of Non-Contact Fingerprinting Devices vs. ContactBased Fingerprinting Devices," Graduate Theses, Dissertations, and Problem Reports, 2017.

[12] D. Deb, T. Chugh, J. Engelsma, K. Cao, N. Nain, J. Kendall and A. K. Jain, "Matching Fingerphotos to Slap Fingerprint Images," arXiv preprint arXiv:1804.08122, 2018.

[13] C. Lin and A. Kumar, "A CNN-Based Framework for Comparison of Contactless to Contact-Based Fingerprints," IEEE Transactions on Information Forensics and Security, vol. 14, no. 3, pp. 662-676, 2019.

[14] A. Dabouei, S. Soleymani, J. Dawson and N. M. Nasrabadi, "Deep Contactless Fingerprint Unwarping," in International Conference on Biometrics, Crete, Greece, 2019.

[15] A. Ross, S. C. Dass and A. K. Jain, "Fingerprint Warping Using Ridge Curve Correspondences," IEEE Transactions on Pattern Analysis and Machine Intelligence, vol. 28, no. 1, pp. 19-30, 2006.

[16] R. Sanchez-Reillo, C. Sanchez-Avila and A. Gonzalez-Marcos, "Biometric Identification Through Hand Geometry Measurements," IEEE Transactions on Pattern Analysis and Machine Intelligence, vol. 22, no. 10, pp. 1168-1171, 2000.

[17] J. Brand and J. S. Mason, "A Comparative Assessment of Three Approaches to Pixel-Level Human Skin-Detection," in 15th International Conference on Pattern Recognition, Barcelona, Spain, 2000.

[18] K. Antonelli, G. Vanderkooy, T. Vlaar and G. Immega, "Fingerprint Image Optical Input apparatus". United States of America Patent US 6259108 B1, 10 July 2001.

[19] S. J. Borza, "Imaging Device, Especially for Optical Fingerprinting". United States of America Patent US 6429927 B1, 6 August 2002.

[20] F. Chen, "3d Fingerprint and Palm Print Data Model and Capture Devices Using Multistructured Lights and Cameras". United States of America Patent US 7609865 B2, 27 October 2009. 
[21] L. G. Hassebrook, D. L. Lau and H. G. Dietz, "System and Method for 3D Imaging Using Structured Light Illumination". United States of America Patent US 8224064 B1, 17 July 2012.

[22] G. Abramovich, K. G. Harding, Q. Hu, S. Manickam, M. Ganesh and C. A. Natis, "Method and System for Contactless Fingerprint Detection and Verification". United States of America Patent US 8406487 B2, 26 March 2013.

[23] N. Engheta, E. N. Pugh Jr., S.-S. Lin and K. M. Yemelyanov, "Polarization and Reflection Based Non-Contact Latent Fingerprint Imaging and Lifting". United States of America Patent US 20070280513 A1, 6 December 2007.

[24] S. Prabhakar and A. K. Jain, "Fingerprint Matching," in Automatic Fingerprint Recognition Systems, New York, NY, Springer Science \& Media, 2004.

[25] A. K. Jain, J. Feng and K. Nandakumar, "Fingerprint Matching," Computer, vol. 43, no. 2, 2010.

[26] M. Adhiyaman and D. Ezhilmaran, "Fingerprint Matching and Similarity Checking System using Minutiae Based Technique," in 2015 IEEE International Conference on Engineering and Technology, Coimbatore, India, 2015.

[27] M. Cappelli, M. Ferrara and D. Maltoni, "Minutiae-Based Fingerprint Matching," in Cross Disciplinary Biometric Systems, Berlin, NY, Springer, 2012.

[28] A. Saleh, A. Bahaa and A. Wahdan, "Fingerprint Recognition," in Advanced Biometric Technologies, Intech, 2011, pp. 201-224.

[29] "Plotting and Interpretating an ROC Curve," [Online]. Available: http://gim.unmc.edu/dxtests/roc2.htm. [Accessed 3 August 2021].

[30] M. E. Schuckers, Computational Metods in Biometric Authentication, Springer, 2010.

[31] "Biometric Quality," NIST, 16 June 2021. [Online]. Available: https://www.nist.gov/programs-projects/biometric-quality. [Accessed 3 August 2021]. 
[32] "NFIQ 2.0," NIST, 2016.

[33] "Skin Color Meter DSM III," Cortex Technology. [Online]. [Accessed March 2021]. 